\title{
Twisted K-theory and Modular Invariants: Quantum Doubles of Finite Groups
}

\author{
David E Evans \\ School of Mathematics, Cardiff University, \\ Senghennydd Road, Cardiff CF24 4YG, Wales, UK. \\ EvansDE@cf.ac.uk
}

Summary. A twisted vector-bundle approach to $\alpha$-induction and modular invariants.

\section{Introduction and statement of results}

The Verlinde algebra is central to conformal field theory and consequently also to the braided subfactor approach to modular invariants. In the braided subfactor approach to modular invariants one has first a factor $N$, which we can take to be type III, and a non-degenerately braided system of endomorphisms ${ }_{N} \mathcal{X}_{N}$ of $N$ whose fusion rules as sectors are precisely those of our Verlinde algebra.

Fixing a braided system of endomorphisms on a type III factor $N$, we look for inclusions $\iota: N \hookrightarrow M$ such that its dual canonical endomorphism $\theta=\bar{\iota} \iota$ decomposes as a sum of endomorphisms from ${ }_{N} \mathcal{X}_{N}$. To produce a modular invariant from such an inclusion, we first employ the Longo-Rehren $\alpha^{ \pm}$-induction method [LR] of extending endomorphisms of $N$ to those in $M$ and then compute the dimensions of the intertwining spaces $Z_{\lambda, \mu}:=\left\langle\alpha_{\lambda}^{+}, \alpha_{\mu}^{-}\right\rangle$. The matrix $Z_{N \subset M}=\left[Z_{\lambda, \mu}\right]$ thus constructed from a braided inclusion $N \subset M$ is a modular invariant [BEK1, E2]. Now we use $\alpha$-induction and the inclusion map $\iota$ to construct finite systems whose general theory has been developed in [BEK1, BEK2]. Let us choose representative endomorphisms of each irreducible subsector of sectors of the form $[\iota \lambda \bar{\imath}], \lambda \in{ }_{N} \mathcal{X}_{N}$. Any subsector of $\left[\alpha_{\lambda}^{+} \alpha_{\mu}^{-}\right]$is automatically a subsector of $[\iota \nu \bar{\iota}]$ for some $\nu$ in ${ }_{N} \mathcal{X}_{N}$ and since we assume the non-degeneracy of the braiding the converse also holds [BEK1]. This set of sectors yields a system ${ }_{M} \mathcal{X}_{M}$ of sectors in general non-commutative (the original sectors from the system ${ }_{N} \mathcal{X}_{N}$ is commutative since it is braided). We define in a similar fashion the chiral systems ${ }_{M} \mathcal{X}_{M}^{ \pm}$to be the subsystems of $\beta \in{ }_{M} \mathcal{X}_{M}$ such that $[\beta]$ is an irreducible subsector of $\left[\alpha_{\lambda}^{ \pm}\right]$. The neutral system ${ }_{M} \mathcal{X}_{M}^{0}$ is defined as the intersection ${ }_{M} \mathcal{X}_{M}^{-} \cap_{M} \mathcal{X}_{M}^{+}$and is non-degenerately braided, so that we obtain ${ }_{M} \mathcal{X}_{M}^{0} \subset{ }_{M} \mathcal{X}_{M}^{ \pm} \subset{ }_{M} \mathcal{X}_{M}$ (see e.g [BE2]). A braided 
subfactor $N \subset M$ producing a modular invariant $Z$ is said to be type I when the dual canonical endomorphism is determined by the vacuum row or column $\oplus Z_{0, \lambda}[\lambda]=\oplus Z_{\lambda, 0}[\lambda]$. In this case, which is equivalent to chiral locality, we can identify both ${ }_{M} \mathcal{X}_{M}^{ \pm}$with ${ }_{N} \mathcal{X}_{M}$ (by $\beta \mapsto \beta \circ \iota, \beta \in{ }_{M} \mathcal{X}_{M}^{ \pm}$if $\iota$ is the inclusion of $N \subset M$.)

There are two cases of interest where there are natural constructions of braided systems or Verlinde algebras. The first is the case of affine Lie algebras or loop groups and their positive energy representations. In this WZW or loop group settings, the modular data ( $S$, and $T$ matrices etc) can be constructed from representation theory of unitary integrable highest weight modules over affine Lie algebras or in exponentiated form from the positive energy representations of loop groups. The subfactor machinery is invoked as follows. Let $L G$ be a loop group (associated to a simple, simply connected loop group $G$ ). Let $L_{I} G$ denote the subgroup of loops which are trivial off some proper interval $I \subset \mathbb{T}$. Then in each level $k$ vacuum representation $\pi_{0}$ of $L G$, we naturally obtain a net of type III factors $\{N(I)\}$ indexed by proper intervals $I \subset \mathbb{T}$ by taking $N(I)=\pi_{0}\left(L_{I} G\right)^{\prime \prime}$ (see [W1, FG, B]). Since the Doplicher-Haag-Roberts DHR selection criterion is met in the (level $k$ ) positive energy representations $\pi_{\lambda}$, there are DHR endomorphisms $\lambda$ naturally associated with them. The rational conformal field theory RCFT modular data matches that in the subfactor setting - in the RCFT Verlinde fusion coincides with the (DHR superselection) sector fusion, i.e. that $N_{\lambda, \mu}^{\nu}=\langle\lambda \mu, \nu\rangle$. The statistics $S$ - and $T$-matrices are identical with the Kac-Peterson $S$ - and $T$-modular matrices which perform the conformal character transformations.

The second is the case of quantum double of finite groups. A given finite system of endomorphisms may not be commutative or even braided but by taking the subfactor analogue of the quantum double of Drinfeld we obtain a subfactor with a non-degenerately braided system of endomorphisms. This construction can in particular be applied to a finite group $G$. This quantum double subfactor is basically the same as the Longo-Rehren inclusion [LR] and is a way of yielding braided systems from not necessarily commutative systems. The modular data from a quantum double subfactor was first established by Ocneanu [EK, Section 12.6] using topological insight, later by Izumi [I1] with an algebraic flavour (see also [SW, KSW]).

Twisted equivariant K-theory [AS] is relevant for both of these settings. Here the equivariant K-theory is twisted by an element of $H^{4}(B G, \mathbb{Z})$. When $G$ is a compact simply connected Lie group, this manifests itself through the equivariant cohomology group $H_{G}^{3}(G, \mathbb{Z})[\mathrm{A}, \mathrm{FHT}, \mathrm{F} 2, \mathrm{~F} 3, \mathrm{~F} 4]$, and for a finite group through $H^{3}(G, \mathbb{T})[\mathrm{F} 1, \mathrm{FQ}]$.

The quantum double of the finite group subfactor $M_{0} \subset M_{0} \rtimes G$, (where the finite group G acts outerly on a type III factor $M_{0}$ ) was identified by Ocneanu and later by Izumi (see [EK]) to be the group-subgroup subfactor $N=M_{0} \rtimes \Delta(G) \subset M_{0} \rtimes(G \times G)=M$ where $\Delta(G)=\{(g, g): g \in G\}$ denotes the diagonal subgroup of $G \times G$. This data can be twisted for every 
$[\omega] \in H^{3}(G, \mathbb{T})[\mathrm{CGP}]$, and the subfactor interpretation of this data is in [I1]. The parameter $[\omega]$ is regarded as the level in this setting [CGP].

It is therefore natural to think in terms of $\Delta(G) \subset G \times G$ and in particular the ambient group $G \times G$ with the diagonal actions. Indeed according to [O1], the module categories of the double are given by pairs $(H, \psi)$ for $H$ a subgroup of $G \times G$ and $\psi$ an arbitrary element of the 2-cohomology group $H^{2}(H, \mathbb{T})$. The corresponding Frobenius algebra or Q-system gives rise to a subfactor $N \subset M$ and hence by [BEK1, E1] produces a modular invariant through the $\alpha$-induction machinery. The ${ }_{N} \mathcal{X}_{M}$ sectors are identified with the $\Delta-H$ bundles or the the equivariant $K$-group $K_{0}^{\Delta \times H}(G \times G)$, and the ${ }_{M} \mathcal{X}_{M}$ sectors with the $H-H$ bundles or the equivariant $K$ - group $K_{0}^{H \times H}(G \times G)$. These identifications of sectors with bundles is compatible with the natural product of sectors and the product of bundles mentioned above.

To translate between equivariant twisted bundles on $G$, for the adjoint action, and equivariant twisted bundles on $G \times G$ with the diagonal action on left and right we need a corresponding cocycle on $G \times G$. If $\omega$ is a 3-cocycle in $Z^{3}(G, \mathbb{T})$, we define the 3-cocycle $\alpha=\pi_{1}^{*} \omega-\pi_{2}^{*} \omega$ on $G \times G$ if $\pi_{1}, \pi_{2}$ are the projections of $G \times G$ on the first and second factors respectively.

In Sect. 2.5, we make precise the relationship between ${ }^{\alpha} K_{0}^{G \times G}(G \times G)$ and ${ }^{\omega} K_{0}^{G}(G)$ where $G \times G$ acts on $G \times G$ by the first factor acting on the left and the second on the right using the diagonal embedding, and $G$ acts $G$ by the adjoint action. The map $(a, b) \rightarrow a b^{-1}$ takes the $G-G$ action to the adjoint $G$ action. This identifies the two K-theories

This work begins the study of understanding $\alpha$-induction and the subfactor approach to modular invariants through twisted equivariant K-theory for the case of quantum doubles of finite groups. This has been thoroughly analysed in [EP2] from the subfactor viewpoint and in [O1] from the viewpoint of module cateogories. ¿From this we should understand the modular invariants which can be realised by subfactors as arising from a subgroup $H$ of $G \times G$ and possible 2-cohomology from $H^{2}(H, \mathbb{T})$. The corresponding full system will be the twisted equivariant K-theory $K_{0}^{H \times H}(G \times G)$, where $H$ acts on the left and right in the natural way. We should identify two homomorphisms $\alpha^{ \pm}$

$$
K_{0}^{\Delta \times \Delta}(G \times G) \rightarrow K_{0}^{H \times H}(G \times G)
$$

whose images commute and generate $K_{0}^{H \times H}(G \times G)$. In the case of a type I pair $(H, \psi)$, where the Q-system satisifies chiral locality (or in categorical language the corresponding Frobenius algebra is commutative), the images are isomorphic to each other and to $K_{0}^{\Delta \times H}(G \times G)$. The neutral system, i.e. the intersection of the images, will not only be commutative, but a nondegenerately braided system - such as $K_{0}^{\Delta(K) \times \Delta(K)}(K \times K)$ for some subgroup $K$ of $G$, i.e. isomorphic to the Verlinde algebra of the quantum double of $K$. For simplification we only discuss this here in the case of the doubles of the finite cyclic groups $\mathbb{Z}_{2}, \mathbb{Z}_{3}$ and the symmetric group $S_{3}$ on three symbols in level zero (i.e. the untwisted case). 


\section{Twisted quantum doubles of finite groups}

\section{$2.1 G$-kernels and 3-cohomology}

Let $G$ be a finite group, and take a $G$-kernel on an infinite factor $M$. That is we have a homomorphism from $G$ into the outer automorphism group Out(M) of $M$, namely the automorphism group Aut(M) of $M$ modulo Int(M) the inner automorphisms of $M$. If $\nu_{g}$ in Aut(M) is a choice of representatives for each $g$ in $G$ of the $G$-kernel, then

$$
\nu_{g} \nu_{h}=\operatorname{Ad}(u(g, h)) \nu_{g h}
$$

for some unitary $u(g, h)$ in $M$, for each pair $g, h$ in $G$. We can assume the normalisation $\nu_{e}=i d_{M}, u(g, e)=u(e, g)=1_{M}$, for all $g$ in $G$, where $e$ is the unit of the group. By associativity of $\nu_{g} \nu_{h} \nu_{k}$, we have a scalar $\omega(g, h, k)$ in $\mathbb{T}$ such that

$$
u(g, h) u(g h, k)=w(g, h, k) \nu_{g}(u(h, k)) u(g, h k),
$$

i.e. $\omega=\partial_{\nu} u$, the $\nu$-coboundary of $u$. A computation [S] shows that $\omega$ is a 3 -cocycle in $Z^{3}(G, \mathbb{T})$ :

$$
\omega(g, h, k) \omega(g, h k, l) \omega(h, k, l)=\omega(g h, k, l) \omega(g, h, k l), \quad g, h, k, l \in G .
$$

Every element of $Z^{3}(G, \mathbb{T}$ ) arises in this way from some $G$-kernel [S, J1] (see also [KT, W2]).

If $\alpha, \beta$ are two endomorphisms between two algebras we let $\operatorname{Hom}(\alpha, \beta)$ denote the intertwiner space $\{x: x \alpha(a)=\beta(a) x, \forall a\}$ in the target algebra. Then $\operatorname{Hom}\left(\nu_{\mathrm{gh}}, \nu_{\mathrm{g}} \nu_{\mathrm{h}}\right)$ is a line bundle $P(g, h)$ spanned by $u(g, h)$.

The conjugate $\bar{\nu}_{g}$ of the automorphism $\nu_{g}$ can be taken to be $\nu_{g^{-1}}$. We define isometries:

$$
r_{g}=u\left(g^{-1}, g\right), \quad \bar{r}_{g}=\bar{\omega}\left(g, g^{-1}, g\right) u\left(g, g^{-1}\right) .
$$

Then $r_{g} \in \operatorname{Hom}\left(1, \overline{\nu_{g}} \nu_{g}\right)$ and $\bar{r}_{g} \in \operatorname{Hom}\left(1, \nu_{g} \overline{\nu_{g}}\right)$ such that

$$
\bar{r}_{g}^{*} \nu_{g}\left(r_{g}\right)=1_{M}, \quad r_{g}^{*} \overline{\nu_{g}}\left(\bar{r}_{g}\right)=1_{M} .
$$

Intertwiners can be written graphically in the notation and conventions of [BEK1]. The set $\left\{\nu_{g}: g \in G\right\}$ of automorphisms forms a system of endomorphisms in the sense of [BEK1], and we can form the quantum double system and consequently the associated topological quantum field theory. The vanishing of the 3 -cohomology class of $\omega$ in $H^{3}(G, \mathbb{T})$ is precisely when we can adjoin unitaries $\left\{v_{g}: g \in G\right\}$ so that $[\mathrm{S}]$ :

$$
v_{g} v_{h}=u(g, h) v_{g h}, \quad \nu_{g}(m)=\operatorname{Ad}\left(v_{g}\right)(m), g, h \in G, m \in M .
$$

In this special case we can form the twisted cross product $M \rtimes G=M \rtimes_{\nu} G$, and then perform the iterated Jones construction

$$
M \subset M \rtimes G \subset M_{1} \subset M_{2} \subset \ldots
$$

and complete to obtain $M_{\infty}$. The quantum double system in this case is the $M_{\infty}-M_{\infty}$ system for the subfactor $A=M \vee M^{\prime} \subset B=M_{\infty}$. 


\subsection{Rectangular algebra}

Ocneanu has introduced tube algebras and double triangle algebras for understanding and handling the combinatorics of intertwiner spaces (see e.g. $[\mathrm{O} 1, \mathrm{O} 2, \mathrm{EK}, \mathrm{BEK} 1])$. We need variants of this - a rectangular algebra and a super-tube algebra. First consider the rectangular algebra $\mathcal{R}=\mathcal{R}^{\omega}(G)$ :

$$
\mathcal{R}^{\omega}(G)=\bigoplus_{a, h, k} \operatorname{Hom}\left(\nu_{h} \nu_{a}, \nu_{h a k} \bar{\nu}_{k}\right)
$$

Note that $\operatorname{Hom}\left(\nu_{h} \nu_{a}, \nu_{b} \bar{\nu}_{k}\right)$ vanishes unless $b=h a k$, when $\operatorname{Hom}\left(\nu_{h} \nu_{a}, \nu_{h a k} \bar{\nu}_{k}\right)$ is one dimensional or a line bundle

$$
R(h, a, k) \simeq P\left(h a k, k^{-1}\right) \otimes P(h, a)^{*},
$$

spanned by the intertwiners

$$
r(h, a, k)=u\left(h a k, k^{-1}\right) u(h, a)^{*} .
$$

There is a natural product map

$$
\begin{gathered}
\operatorname{Hom}\left(\nu_{h^{\prime}} \nu_{a^{\prime}}, \nu_{h^{\prime} a^{\prime} k^{\prime}} \bar{\nu}_{k^{\prime}}\right) \times \operatorname{Hom}\left(\nu_{h} \nu_{a}, \nu_{h a k} \bar{\nu}_{k}\right) \\
\rightarrow \operatorname{Hom}\left(\nu_{h^{\prime} h} \nu_{a}, \nu_{h^{\prime} h a k k^{\prime}} \bar{\nu}_{k k^{\prime}}\right)
\end{gathered}
$$

given by

$$
S^{\prime} \times S \rightarrow \delta_{a^{\prime}, h a k} \nu_{h^{\prime}}\left(S^{\prime}\right) S,
$$

so that we have the coherence:

$$
R\left(h^{\prime}, h a k, k^{\prime}\right) \otimes R(h, a, k) \simeq R\left(h^{\prime} h, a, k k^{\prime}\right)
$$

In addition, there is an involution

$$
\begin{aligned}
\operatorname{Hom}\left(\nu_{\mathrm{h}} \nu_{\mathrm{a}}, \nu_{\mathrm{hak}} \bar{\nu}_{\mathrm{k}}\right) & \rightarrow \operatorname{Hom}\left(\nu_{h a k} \bar{\nu}_{k}, \nu_{h} \nu_{a},\right) \rightarrow \operatorname{Hom}\left(\bar{\nu}_{h} \nu_{h a k}, \nu_{a} \nu_{k}\right) \\
S & \rightarrow S^{\dagger}=r_{h}^{*} \bar{\nu}_{h}\left[S^{*} \nu_{h a k}\left(\bar{r}_{k^{-1}}\right)\right],
\end{aligned}
$$

obtained by first taking the involution in the von Neumann algebra $M$ and then using Frobenius reciprocities [BEK1]. Here to avoid confusion we denote * as the involution in the algebra $M$, and $\dagger$ as the involution in the rectangular space. Conseqently,

$$
R(h, a, k)^{\dagger} \simeq R\left(h^{-1}, h a k, k^{-1}\right) .
$$

It is this involution that we will use to get a $*$-structure on our algebras and when there is no likely confusion we will denote this by $*$ as usual.

However, there is another related conjugate linear automorphism. First, note that there are natural identifications of intertwiner spaces 


$$
\begin{gathered}
\operatorname{Hom}\left(\nu_{h} \nu_{b}, \nu_{h b k} \bar{\nu}_{k}\right) \rightarrow \operatorname{Hom}\left(\nu_{h b k} \bar{\nu}_{k}, \nu_{h} \nu_{b}\right) \rightarrow \operatorname{Hom}\left(\bar{\nu}_{k} \bar{\nu}_{b}, \bar{\nu}_{h b k} \nu_{h}\right) \\
\left.S \rightarrow S^{b}=\bar{\nu}_{h a k}\left[\nu_{h}\left(\bar{r}_{a}^{*}\right) S^{*}\right)\right] r_{h a k},
\end{gathered}
$$

by first taking adjoints and then using Frobenius reciprocity. This is a conjugate linear automorphism so that

$$
R(h, a, k)^{b} \simeq R\left(k^{-1}, a^{-1}, h^{-1}\right) .
$$

This endows $\mathcal{R}^{\omega}(G)$ as a finite dimensional $C^{*}$-algebra. In terms of the canonical generators we have the relations:

$$
\begin{aligned}
r\left(h^{\prime}, a^{\prime}, k^{\prime}\right) r(h, a, k)= & \delta_{a^{\prime}, h a k} \omega\left(h^{\prime}, h, a\right) \bar{\omega}\left(h^{\prime}, h a k, k^{-1}\right) \\
& \times \omega\left(h^{\prime} h a k k^{\prime}, k^{-1}, k^{-1}\right) r\left(h^{\prime} h, a, k k^{\prime}\right), \\
r(h, a, k)^{*}= & \bar{\omega}\left(h^{-1}, h, a\right) \omega\left(h^{-1}, h a k, k^{-1}\right) \\
& \times \bar{\omega}\left(a, k, k^{-1}\right) r\left(h^{-1}, h a k, k^{-1}\right), \\
r(h, a, k)^{b}= & \omega\left(k^{-1}, a, a^{-1}\right) \omega\left(k^{-1} a^{-1} h^{-1}, h a k, k^{-1}\right) \\
& \times \quad \bar{\omega}\left(k^{-1} a^{-1} h^{-1}, h, a\right) r\left(k^{-1}, a^{-1}, h^{-1}\right) .
\end{aligned}
$$

\subsection{Tube algebra}

We will use this rectangular space to construct the tube algebra and the supertube algebra. First the tube algebra is the space of intertwiners $\mathcal{D}=\mathcal{D}^{\omega}(G)$ :

$$
\mathcal{D}^{\omega}(G)=\bigoplus_{a, h} \operatorname{Hom}\left(\nu_{h} \nu_{a}, \nu_{h a h^{-1}} \nu_{h}\right) .
$$

Here $\operatorname{Hom}\left(\nu_{h} \nu_{a}, \nu_{h a h^{-1}} \nu_{h}\right)$ is one dimensional or a line bundle

$$
C(a, h) \simeq P\left(h a h^{-1}, h\right) \otimes P(h, a)^{*},
$$

spanned by the intertwiners

$$
c(a, h)=r\left(h, a, h^{-1}\right)=u\left(h a h^{-1}, h\right) u(h, a)^{*} .
$$

We have the coherence and involutive properties:

$$
\begin{array}{r}
C\left(h a h^{-1}, h^{\prime}\right) \otimes C(a, h) \simeq C\left(a, h^{\prime} h\right), \\
C(a, h)^{\dagger} \simeq C\left(h a h^{-1}, h^{-1}\right),
\end{array}
$$

The tube algebra is then a finite dimensional $C^{*}$-algebra with generators $\{c(a, h): a, h\}$ and relations:

$$
\begin{aligned}
c\left(a^{\prime}, h^{\prime}\right) c(a, h)= & \delta_{a^{\prime}, h a h^{-1}} w\left(h^{\prime}, h, a\right) \bar{w}\left(h^{\prime}, h a h^{-1}, h\right) \\
& \times w\left(h^{\prime} h a h^{-1} h^{\prime-1}, h^{\prime}, h\right) c\left(a, h^{\prime} h\right), \\
c(a, h)^{*}= & \bar{w}\left(h^{-1}, h, a\right) w\left(h^{-1}, h a h^{-1}, h\right) \\
& \times \bar{w}\left(a, h^{-1}, h\right) c\left(h a h^{-1}, h^{-1}\right) .
\end{aligned}
$$


The group $G$ has two parent algebras. One is the function algebra $C(G)$ of complex valued functions on $G$ under pointwise multiplication $f g(a)=$ $f(a) g(a)$ and co-multiplication $\Delta(f)(a, b)=f(a b)$, spanned by the delta functions $\delta_{g}(h)=\delta_{g, h}$. The other is its dual $C(G)^{*}$ as a Hopf algebra, the group algebra $\mathbb{C}(G)$ with multiplication $a \otimes b \rightarrow a b$ and co-multiplication $a \rightarrow a \otimes a$. The function algebra $C(G)$ and group algebra $\mathbb{C}(G)$ embedd into the tube algebra as $\delta_{g} \rightarrow c(g, e)$, and $h \rightarrow c(e, h)$, so that indeed the tube algebra is their tensor product $C(G) \otimes \mathbb{C}(G)$ as a vector space, with $c(g, h)$ identified with $\delta_{g} \otimes h$, but the product on $\mathcal{D}^{\omega}(G)$ is twisted by the 3-cocycle $\omega$. The representations of the tube algebra $\mathcal{D}^{\omega}(G)$ is described by $G$-equivariant vector bundles over $G$. If $\rho$ is a representation of $\mathcal{D}=\mathcal{D}^{\omega}(G)$ on $V$, then since it is in particular a representation of the function algebra $C(G)$, we can write $V_{g}=\rho\left(\delta_{g}\right) V$ to give a vector bundle over $G$.

We can read the coherence or the $\mathcal{D}^{\omega}(G)$-action on $\mathrm{V}$ as $G$-equivariance expressed as

$$
C(a, h) \otimes V_{a} \simeq V_{h a h^{-1}},
$$

where $C(a, h)$ is the line bundle $\operatorname{Hom}\left(\nu_{h} \nu_{a}, \nu_{h a h^{-1}} \nu_{h}\right)$, so that we have maps

$$
\begin{gathered}
\pi_{h}^{V}: C(a, h) \otimes V_{a} \rightarrow V_{h a h^{-1}} \\
\pi_{h}^{V}\left(\ell \otimes v_{a}\right)=\rho(\ell) v_{a} .
\end{gathered}
$$

By some abuse of notation we find it convenient to write this as

$$
h . v_{a}=\pi_{h}^{V}\left(c \otimes v_{a}\right)=\rho(c) v_{a},
$$

for the particular interwiner $\ell=c(a, h)$ or cross section as in Eq. (18). Thus $h . v_{a} \in V_{h a h^{-1}}$, for $v_{a} \in V_{a}$. We can think of this as one vector space $V_{a}$ sitting over one end of the tube and $V_{h a h^{-1}}$ over the other, with one transported to the other via the line bundle $C(a, h)$. Note that the coherence Eq. (19) of line bundles is reflected as a twisted left action:

$$
h^{\prime} .\left(h \cdot v_{a}\right)=\omega\left(h^{\prime}, h, a\right) \bar{\omega}\left(h^{\prime}, h a h^{-1}, h\right) \omega\left(h^{\prime} h a h^{-1} h^{\prime-1}, h^{\prime}, h\right) h^{\prime} h . v_{a} .
$$

Elements of $\operatorname{Rep}\left(\mathcal{D}^{\omega}(\mathrm{G})\right)$, the representations of $\mathcal{D}^{\omega}(G)$, are thus described as vector bundles over $G$, with a twisted left action satisfying Eq. (24).

To take a fusion product of two representations of $\mathcal{D}=\mathcal{D}^{\omega}(G)$, we need the co-multiplication operator $\Delta$ from $\mathcal{D} \rightarrow \mathcal{D} \otimes \mathcal{D}$, which is obtained as follows:

$$
\begin{aligned}
\Delta c(a, h)=\sum_{a^{\prime}, a^{\prime \prime}: a^{\prime} a^{\prime \prime}=a} \omega\left(h, a^{\prime}, a^{\prime \prime}\right) \omega\left(h a^{\prime} h^{-1}, h a^{\prime \prime} h^{-1}, h\right) \\
\times \bar{\omega}\left(h a^{\prime} h^{-1}, h, a^{\prime \prime}\right) c\left(a^{\prime}, h\right) \otimes c\left(a^{\prime \prime}, h\right) .
\end{aligned}
$$

Suppose $\rho_{V^{1}}$ and $\rho_{V^{2}}$ are representations of $\mathcal{D}^{\omega}(G)$ on $V^{1}$ and $V^{2}$ respectively, then we can define the fusion product representation $\rho_{V^{1}} \otimes \rho_{V^{2}}$ on $V^{1} \otimes V^{2}$ by: 


$$
\left(\rho_{V^{1}} \otimes \rho_{V^{2}}\right)(x)=\left(\rho_{V^{1}} \otimes \rho_{V^{2}}\right) \Delta(x), x \in \mathcal{D}^{\omega}(G) .
$$

This can be interpreted as a product on $G$-equivariant vector bundles, using

$$
\Delta c(a, e)=\sum_{a^{\prime}, a^{\prime \prime}: a^{\prime} a^{\prime \prime}=a} c\left(a^{\prime}, e\right) \otimes c\left(a^{\prime \prime}, e\right) .
$$

We form the vector bundle $V^{1} \otimes V^{2}$ by

$$
\begin{aligned}
\left(V^{1} \otimes V^{2}\right)_{a} & =\left(\rho_{V^{1}} \otimes \rho_{V^{2}}\right)(c(a, e))\left(V^{1} \otimes V^{2}\right) \\
& =\left(\rho_{V^{1}} \otimes \rho_{V^{2}}\right) \Delta(c(a, e))\left(V^{1} \otimes V^{2}\right)=\oplus_{a^{\prime} a^{\prime \prime}=a} V_{a^{\prime}}^{1} \otimes V_{a^{\prime \prime}}^{2} .
\end{aligned}
$$

The $G$-action on $V^{1} \otimes V^{2}$ is then expressed as

$$
\begin{aligned}
h .\left(v_{a^{\prime}}^{1} \otimes v_{a^{\prime \prime}}^{2}\right)= & \omega\left(h, a^{\prime}, a^{\prime \prime}\right) \omega\left(h a^{\prime} h^{-1}, h a^{\prime \prime} h^{-1}, h\right) \\
& \times \bar{\omega}\left(h a^{\prime} h^{-1}, h, a^{\prime \prime}\right) h \cdot v_{a^{\prime}}^{1} \otimes h \cdot v_{a^{\prime \prime}}^{2},
\end{aligned}
$$

for $v^{1} \in V^{1}, v^{2} \in V^{2}$.

The trivial bundle $V_{a}^{0}=\delta_{a, e} \mathbb{C}$, with trivial action, defines a representation or equivariant bundle $V^{0}$ so that $V^{0} \otimes V^{1} \simeq V^{1} \simeq V^{1} \otimes V^{0}$ for any other bundle $V^{1}$.

The tube algebra $\mathcal{D}^{\omega}(G)$ is a (quasi-associative) Hopf algebra with $R$ matrix:

$$
R=\sum_{a^{\prime}, a^{\prime \prime}} c\left(a^{\prime}, e\right) \otimes c\left(a^{\prime \prime}, a^{\prime}\right) .
$$

Then the braiding operator is the isomorphism

$$
\varepsilon\left(V^{1}, V^{2}\right)=\tau\left(\rho_{V^{1}} \otimes \rho_{V^{2}}\right)(R): V^{1} \otimes V^{2} \rightarrow V^{2} \otimes V^{1},
$$

where $\tau$ is the transposition from $V^{1} \otimes V^{2}$ to $V^{2} \otimes V^{1}$. In terms of vector bundles, this braiding takes the form:

$$
\varepsilon\left(V^{1}, V^{2}\right)\left[v_{a^{\prime}}^{1} \otimes v_{a^{\prime \prime}}^{2}\right]=a^{\prime} \cdot v_{a^{\prime \prime}}^{2} \otimes v_{a^{\prime}}^{1}
$$

for $v^{1} \in V^{1}, v^{2} \in V^{2}$, and is a $G$-equivariant bundle isomorphism.

This is one picture of the twisted quantum double of Drinfeld of the finite group [AC, ACM, DPR, I1, M] . It is more convenient for us look at this from another persepective starting in the next section.

\subsection{Super-tube algebra and $\Delta-\Delta$ equivariant bundles}

We want to switch from $G$-equivariant vector bundles on $G$ to equivariant vector bundles over $G \times G$. For this we consider the super-tube algebra $\mathcal{E}=$ $\mathcal{E}^{\omega}(G)$, defined as the following finite dimensional $C^{*}$-algebra. First note that there are natural identifications of intertwiner spaces

$$
\operatorname{Hom}\left(\nu_{h} \nu_{b}, \nu_{h b k} \bar{\nu}_{k}\right) \rightarrow \operatorname{Hom}\left(\nu_{h b k} \bar{\nu}_{k}, \nu_{h} \nu_{b}\right) \rightarrow \operatorname{Hom}\left(\bar{\nu}_{k} \bar{\nu}_{b}, \bar{\nu}_{h b k} \nu_{h}\right)
$$


by first taking adjoints and then using Frobenius reciprocity [BEK1]. Let $S \rightarrow S^{\text {b }}$ denote the composition of these identifications. We then combine to form the map

$$
\operatorname{Hom}\left(\nu_{h} \nu_{a}, \nu_{h a k} \bar{\nu}_{k}\right) \times \operatorname{Hom}\left(\nu_{h} \nu_{b}, \nu_{h b k} \bar{\nu}_{k}\right) \rightarrow \operatorname{Hom}\left(\nu_{h} \nu_{a} \bar{\nu}_{b}, \nu_{h a k} \bar{\nu}_{h b k} \nu_{h}\right)
$$

where

$$
(T, S) \rightarrow \nu_{h a k}\left(S^{b}\right) T .
$$

We denote this intertwiner by $T \times \bar{S}$. Furthermore there is an involution which takes the intertwiner $T \times \bar{S}$ to $T^{\dagger} \times \overline{S^{\dagger}}$.

The interwiner space

$$
T(h,(a, b), k)=\operatorname{Hom}\left(\nu_{h} \nu_{a} \bar{\nu}_{b}, \nu_{h a k} \bar{\nu}_{h b k} \nu_{h}\right) \simeq R(h, a, k) \otimes R(h, b, k)^{*}
$$

has generators

$$
\begin{aligned}
t(h,(a, b), k) & =\nu_{h a k}\left(r(h, b, k)^{b}\right) r(h, a, k) \\
& =[r(h, a, k)] \times[r(h, b, k)]^{-} .
\end{aligned}
$$

We have the coherence and involutive properties:

$$
\begin{array}{r}
T\left(h^{\prime}, h a k, k^{\prime}\right) \otimes T\left(h^{\prime}, a, k\right) \simeq T\left(h^{\prime} h, a, k k^{\prime}\right), \\
T(h, a, k)^{\dagger} \simeq T\left(k^{-1}, h a k, h^{-1}\right) .
\end{array}
$$

Consequently, the super-tube algebra $\mathcal{E}=\mathcal{E}^{\omega}(G)$ defined as

$$
\mathcal{E}=\mathcal{E}^{\omega}(G)=\bigoplus_{a, b, h, k} \operatorname{Hom}\left(\nu_{h} \nu_{a} \bar{\nu}_{b}, \nu_{h a k} \bar{\nu}_{h b k} \nu_{h}\right),
$$

is a finite dimensional $C^{*}$-algebra, with generators $\{t(h,(a, b), k): h, a, b, k \in$ $G\}$ and relations :

$$
\begin{gathered}
t\left(h^{\prime}, x^{\prime}, k^{\prime}\right) t(h, x, k)=\delta_{x^{\prime}, h x k} \alpha\left(h^{\prime}, h, x\right) \bar{\alpha}\left(h^{\prime}, h x k, k^{-1}\right) \\
\times \quad \alpha\left(h^{\prime} h x k k^{\prime}, k^{\prime-1}, k^{-1}\right) t\left(h^{\prime} h x k k^{\prime}, k^{\prime-1}, k^{-1}\right), \\
t(h, x, k)^{\dagger}=\bar{\alpha}\left(h^{-1}, h, x\right) \alpha\left(h^{-1}, h x k, k^{-1}\right) \\
\times \bar{\alpha}\left(x, k, k^{-1}\right) t\left(h^{-1}, h x k, k^{-1}\right) .
\end{gathered}
$$

for $x, y$ in $\Gamma=G \times G$ and $h, k$ in $\Delta(G)$, the diagonal subgroup identified with $G$. Here if $\omega$ is a 3 -cocycle in $Z^{3}(G, \mathbb{T})$, we define the 3-cocycle $\alpha=\pi_{1}^{*} \omega-\pi_{2}^{*} \omega$ on $\Gamma$ if $\pi_{1}, \pi_{2}$ are the projections of $G \times G$ on the first and second factors respectively.

We now consider $\operatorname{Rep}\left(\mathcal{E}^{\omega}(\mathrm{G})\right)$, the representations of $\mathcal{E}^{\omega}(G)$. We claim that these are described by $\Delta-\Delta$ equivariant twisted vector bundles over $G \times G$. The function algebra on $G \times G$ embedds in $\mathcal{E}^{\omega}(G)$ by $\delta_{x} \rightarrow t(e, x, e), \quad x \in G \times G$. Thus if $\rho$ is a representation of $\mathcal{E}^{\omega}(G)$ on $W$, it is in particular a representation of the function algebra and so we can write 


$$
W_{x}=\rho\left(\delta_{x}\right) W, \quad x \in G \times G,
$$

to get a vector bundle over $G \times G$. The $\Delta-\Delta$ equivariance is expressed as

$$
T(h, x, k) \otimes W_{x} \simeq W_{h x k}, \quad x \in G \times G, \quad h, k \in \Delta(G) .
$$

In other words, we can act with the diagonal subgroup $G=\Delta(G)$ on the left and right as

$$
\begin{gathered}
h . v_{x}=t(h, x, e) v_{x}, \\
v_{x} . k=t\left(e, x k, k^{-1}\right)^{*} v_{x} .
\end{gathered}
$$

So for $v_{x} \in W_{x}$, we have $h . v_{x} \in W_{h x}, v_{x} . k \in W_{x k}$. Then we have the projective relations:

$$
\begin{aligned}
h^{\prime} \cdot\left(h \cdot v_{x}\right) & =\alpha\left(h^{\prime}, h, x\right) h^{\prime} h \cdot v_{x}, \\
\left(v_{x} \cdot k\right) . k^{\prime} & =\bar{\alpha}\left(x, k, k^{\prime}\right) v_{x} \cdot k k^{\prime} .
\end{aligned}
$$

Note that the left and right actions do not in general commute but

$$
h .\left(v_{x} . k\right)=\alpha(h, x, k)\left(h . v_{x}\right) . k,
$$

as a consequence of the super-tube relation:

$$
t(h, x k, e) t\left(e, x k, k^{-1}\right)^{*}=\alpha(h, x, k) t\left(e, h x k, k^{-1}\right)^{*} t(h, x, e) .
$$

To take the fusion product of two representations of $\operatorname{Rep}\left(\mathcal{E}^{\omega}(\mathrm{G})\right)$, we need the co-multiplication operator $\Delta$ from $\mathcal{E} \rightarrow \mathcal{E} \otimes \mathcal{E}$. The co-multiplication is given by:

$$
\begin{aligned}
\Delta t(h, x, k)= & \sum_{l, x^{\prime} x^{\prime \prime}=x} \alpha\left(h, x^{\prime}, x^{\prime \prime}\right) \alpha\left(h x^{\prime} l, l^{-1} x^{\prime \prime} k, k^{-1}\right) \\
& \times \bar{\alpha}\left(h x^{\prime} l, l^{-1}, x^{\prime \prime}\right) t\left(h, x^{\prime}, l\right) \otimes t\left(l^{-1}, x^{\prime \prime}, k\right) .
\end{aligned}
$$

Suppose $\rho_{W^{1}}$ and $\rho_{W^{2}}$ are representations of $\mathcal{E}^{\omega}(G)$ on $W^{1}$ and $W^{2}$ respectively, then we can define the fusion product representation $\rho_{W^{1}} \otimes \rho_{W^{2}}$ on $W^{1} \otimes W^{2}$ by:

$$
\left(\rho_{W^{1}} \otimes \rho_{W^{2}}\right)(x)=\left(\rho_{W^{1}} \otimes \rho_{W^{1}}\right) \Delta(x), x \in \mathcal{E}^{\omega}(G)
$$

This can be interpreted as a product on $\Delta$ - $\Delta$-equivariant vector bundles on $G \times G$, using

$$
\Delta t(e, x, e)=\sum_{l, x^{\prime} x^{\prime \prime}=x} \bar{\alpha}\left(h x^{\prime} l, l^{-1}, x^{\prime \prime}\right) t\left(e, x^{\prime}, l\right) \otimes t\left(l^{-1}, x^{\prime \prime}, e\right)
$$

The bundle is given by

$$
\left(W^{1} \otimes W^{2}\right)_{x}=\left(\rho_{W^{1}} \otimes \rho_{W^{2}}\right) \Delta(t(e, x, e))\left(W^{1} \otimes W^{2}\right)=\oplus_{x^{\prime} x^{\prime \prime}=x} W_{x^{\prime}}^{1} \otimes W_{x^{\prime \prime}}^{2},
$$


where we must identify according to the equivalence relation:

$$
v_{x^{\prime}} . l \otimes w_{x^{\prime \prime}}=\alpha\left(x^{\prime}, l, x^{\prime \prime}\right) v_{x^{\prime}} \otimes l . w_{x^{\prime \prime}} .
$$

Then the $\Delta-\Delta$ action on $W^{1} \otimes W^{2}$ is given by

$$
\begin{aligned}
& \Delta t(h, x, e)=\sum_{l, x^{\prime} x^{\prime \prime}=x} \alpha\left(h, x^{\prime}, x^{\prime \prime}\right) \bar{\alpha}\left(h x^{\prime} l, l^{-1}, x^{\prime \prime}\right) t\left(h, x^{\prime}, l\right) \otimes t\left(l^{-1}, x^{\prime \prime}, e\right) \\
& \Delta t(e, x, k)=\sum_{l, x^{\prime} x^{\prime \prime}=x} \alpha\left(x^{\prime} l, l^{-1} x^{\prime \prime} k, k^{-1}\right) \bar{\alpha}\left(x^{\prime} l, l^{-1}, x^{\prime \prime}\right) \\
& \times \quad t\left(e, x^{\prime}, l\right) \otimes t\left(l^{-1}, x^{\prime \prime}, k\right) .
\end{aligned}
$$

Thus the diagonal group $\Delta$ acts on the left and right of $W^{1} \otimes W^{2}$ by

$$
\begin{aligned}
& h .\left(v_{x^{\prime}}^{1} \otimes v_{x^{\prime \prime}}^{2}\right)=\alpha\left(h, x^{\prime}, x^{\prime \prime}\right) h . v_{x^{\prime}}^{1} \otimes v_{x^{\prime \prime}}^{2}, \\
& \left(v_{x^{\prime}}^{1} \otimes v_{x^{\prime \prime}}^{2}\right) . k=\bar{\alpha}\left(x^{\prime}, x^{\prime \prime}, k\right) v_{x^{\prime}}^{1} \otimes v_{x^{\prime \prime}}^{2} \cdot k .
\end{aligned}
$$

The trivial bundle $W_{x}^{0}=\delta_{x, e} \mathbb{C}$, with trivial action, defines a representation or equivariant bundle $W^{0}$ so that $W^{0} \otimes W^{1} \simeq W^{1} \simeq W^{1} \otimes W^{0}$ for any other bundle $W^{1}$.

\subsection{Morita equivalence of $G$ equivariant and $\Delta-\Delta$ equivariant bundles}

We relate the tube algebra $\mathcal{D}$ and the super-tube algebra $\mathcal{E}$ via a Morita equivalence implemented by an intermediary $\mathcal{D}-\mathcal{E}$ bimodule $\mathcal{V}$ which we define as

$$
\mathcal{V}^{\omega}(G)=\bigoplus_{a, b, h} \operatorname{Hom}\left(\nu_{h} \nu_{a} \bar{\nu}_{b}, \nu_{h a b^{-1} h^{-1}} \nu_{h}\right) .
$$

We can embedd elements of the tube algebra $\mathcal{D}$ in the super-tube algebra $\mathcal{E}$ by

$$
\operatorname{Hom}\left(\nu_{h} \nu_{a}, \nu_{h a h^{-1}} \nu_{h}\right) \rightarrow \operatorname{Hom}\left(\nu_{h} \nu_{a} \bar{\nu}_{e}, \nu_{h a h^{-1}} \bar{\nu}_{e} \nu_{h}\right)
$$

by $S \rightarrow S \times c(e, h)^{-}$, if $S \in \operatorname{Hom}\left(\nu_{h} \nu_{a}, \nu_{h a h^{-1}} \nu_{h}\right)$, so that we identify $c(a, h)$ and $t(h,(a, e), h)$.

Similarly, we can regard an element of the space $\mathcal{V}$ as an element of the super-tube algebra $\mathcal{E}$ by identifying

$$
\operatorname{Hom}\left(\nu_{h} \nu_{a}, \nu_{h a b^{-1} h^{-1}} \nu_{h b}\right) \times \operatorname{Hom}\left(\nu_{h b} \bar{\nu}_{b}, \nu_{h}\right)=\operatorname{Hom}\left(\nu_{h} \nu_{a} \bar{\nu}_{b}, \nu_{h a b^{-1} h^{-1}} \nu_{h}\right)
$$

in $\mathcal{V}$ with

$$
\operatorname{Hom}\left(\nu_{h} \nu_{a}, \nu_{h a k} \bar{\nu}_{k}\right) \times \operatorname{Hom}\left(\nu_{h} \nu_{b}, \nu_{h b k} \bar{\nu}_{k}\right)=\operatorname{Hom}\left(\nu_{h} \nu_{a} \bar{\nu}_{b}, \nu_{h a k} \bar{\nu}_{h b k} \nu_{h}\right)
$$

in $\mathcal{E}$, for $k=(h b)^{-1}$. Then using the product in the super-tube algebra $\mathcal{E}$, we can regard $\mathcal{V}$ as a $\mathcal{D}-\mathcal{E}$ bimodule. This space $\mathcal{V}$ has generators 


$$
v((a, b), h)=t\left(h,(a, b),(h b)^{-1}\right), \quad h, a, b \in G,
$$

with the $\mathcal{D}-\mathcal{E}$ bimodule relations:

$$
\begin{array}{r}
v\left(x^{\prime}, h^{\prime}\right) t(h, x, k)=\delta_{x^{\prime}, h x k} \alpha\left(h^{\prime}, h, x\right) \alpha\left(h^{\prime} h x k\left(h^{\prime} h b k\right)^{-1}, h^{\prime} h b k, k^{-1}\right) \\
\times \bar{\alpha}(h, h x k, k) v\left(x, h^{\prime} h\right), \\
c\left(x^{\prime}, h^{\prime}\right) v(x, h)=\delta_{x^{\prime}, h a b^{-1} h^{-1}} \alpha\left(h^{\prime}, h, x\right) \alpha\left(h^{\prime} h x\left(h^{\prime} h b\right)^{-1}, h^{\prime}, h b\right) \\
\times \bar{\alpha}\left(h^{\prime}, h x(h b)^{-1}, h b\right) v\left(x, h^{\prime} h\right),
\end{array}
$$

for $x=(a, b)$.

Thus if $W$ is an $\mathcal{E}$ module, we can form $\mathcal{V} \otimes_{\mathcal{E}} W$ as a natural $\mathcal{D}$ module, and if $V$ is an $\mathcal{D}$ module, we can form $\mathcal{V}^{*} \otimes_{\mathcal{D}} V$ as a natural $\mathcal{E}$ module. This gives an equivalence of $\mathcal{D}$ and $\mathcal{E}$-modules or a correspondence between $G$-equivariant vector bundles on $G$ and $\Delta$ - $\Delta$ equivariant vector bundles on $G \times G$. Let us look at this in more detail at the level of vector bundles. If $V$ is a $\mathcal{D}$-module or a $G$-equivariant twisted vector bundle, then we form a vector bundle over $G \times G$ by:

$$
W_{a, b}=P\left(a, b^{-1}\right) \otimes V_{a b^{-1}},
$$

where $P$ is the vector bundle $P\left(a, b^{-1}\right)=\operatorname{Hom}\left(\nu_{\mathrm{ab}^{-1}}, \nu_{\mathrm{a}} \overline{\nu_{\mathrm{b}}}\right)$, as before. Then $W$ becomes an $\mathcal{E}$-module by the coherent actions:

$$
\begin{aligned}
T(h,(a, b), k) \otimes W_{a, b} & \simeq T(h,(a, b), k) \otimes P\left(a, b^{-1}\right) \otimes V_{a b^{-1}} \\
& \simeq P\left(h a k,(h b k)^{-1}\right) \otimes C\left(a b^{-1}, h\right) \otimes V_{a b^{-1}} \\
& \simeq P\left(h a k,(h b k)^{-1}\right) \otimes V_{h a b^{-1} h^{-1}} \simeq W_{h a k^{-1}, h b k^{-1}} .
\end{aligned}
$$

In particular if $v \in V$, we define $w \in W$ by

$$
w_{a, b}=v((a, b), e)^{*} \otimes v_{a b^{-1}} .
$$

Then $W$ becomes a $\Delta-\Delta$ equivariant twisted bundle by the actions:

$$
\begin{array}{r}
h . w_{a, b}=\alpha\left(h^{-1}, h, x\right) \bar{\alpha}\left(x b^{-1}, h^{-1}, h b\right) \alpha\left(h^{-1}, h x b^{-1}, h b\right) \\
\times \quad v(h(a, b), e) \otimes h \cdot v_{a b^{-1}}, \\
w_{a, b} . k=\bar{\omega}\left(a b^{-1}, b, k^{-1}\right) v((a, b) k, e) \otimes v_{a b^{-1}} .
\end{array}
$$

Conversely, suppose that $W$ is an $\mathcal{E}$-module or an equivariant $\Delta$ - $\Delta$ twisted bundle over $G \times G$, then we can form a bundle $V$ over $G$ by:

$$
V_{d}=\oplus_{a b^{-1}=d} P\left(a, b^{-1}\right)^{*} \otimes W_{a, b} .
$$

This becomes a $\mathcal{D}$ module by the coherence:

$$
\begin{aligned}
C(d, h) \otimes V_{d} & \simeq \oplus_{a b^{-1}=d} C(d, h) \otimes P\left(a, b^{-1}\right)^{*} \otimes W_{a, b} \\
& \simeq \oplus_{a b^{-1}=d} P\left(h a,(h b)^{-1}\right)^{*} \otimes W_{h a, h b} \simeq V_{h d h^{-1}} .
\end{aligned}
$$

In particular if $w \in W$, we define $v \in V$ by 


$$
v_{d}=\oplus_{a b^{-1}=d} v((a, b), e) \otimes w_{a, b} .
$$

Then $V$ becomes a $G$ equivariant twisted vector bundle over $G$ by

$$
h . v_{d}=\oplus_{a b^{-1}=d} \bar{\omega}\left(h, a b^{-1}, b\right) \omega\left(h a b^{-1} h^{-1}, h, b\right) v(h(a, b), e) \otimes h . w_{a, b} .
$$

This gives a (Morita) equivalence of $\operatorname{Rep}\left(\mathcal{D}^{\omega}(G)\right)$ and $\operatorname{Rep}\left(\mathcal{E}^{\omega}(G)\right)$, because $\mathcal{V} \otimes_{\mathcal{E}} \mathcal{V}^{*}=\mathcal{D}$ and $\mathcal{V}^{*} \otimes_{\mathcal{D}} \mathcal{V}=\mathcal{E}$, cf $[\mathrm{R}]$

The multiplicative properties of these equivalences are as follows. Suppose $V^{1}, V^{2}$ are $\mathcal{D}$ modules. Then we form the fusion product module $V=V^{1} \otimes V^{2}$, and the corresponding $\mathcal{E}$ modules, $W^{1}=\mathcal{V}^{*} \otimes_{\mathcal{D}} V^{1}, W^{2}=\mathcal{V}^{*} \otimes_{\mathcal{D}} V^{1}$ and $W=\mathcal{V}^{*} \otimes_{\mathcal{D}} V$ respectively. Then

$$
\begin{aligned}
W_{a, b} & \simeq P\left(a, b^{-1}\right) \otimes V_{a b^{-1}} \simeq P\left(a, b^{-1}\right) \otimes\left(V^{1} \otimes V^{2}\right)_{a b^{-1}} \\
& \simeq \oplus_{a b^{-1}=x y^{-1}} P\left(a, b^{-1}\right) \otimes P\left(x, y^{-1}\right)^{*} \otimes V_{x}^{1} \otimes V_{y^{-1}}^{2} \\
& \simeq \oplus_{a=a^{\prime} a^{\prime \prime}} P\left(a^{\prime}, a^{\prime \prime}\right)^{*} \otimes P\left(a^{\prime \prime}, b^{-1}\right) \otimes V_{a^{\prime}}^{1} \otimes V_{a^{\prime \prime} b^{-1}}^{2} \\
& \simeq \oplus_{a=a^{\prime} a^{\prime \prime}} P\left(a^{\prime}, a^{\prime \prime}\right)^{*} \otimes W_{a^{\prime}, e}^{1} \otimes W_{a^{\prime \prime}, b}^{2} \simeq\left(W^{1} \otimes W^{2}\right)_{a, b},
\end{aligned}
$$

so that $W \simeq W^{1} \otimes W^{2}$.

On the other hand suppose that $W^{1}, W^{2}$ are $\mathcal{E}$ modules. Then we form the fusion product module $W=W^{1} \otimes W^{2}$, and the corresponding $\mathcal{D}$ modules, $V^{1}=\mathcal{V} \otimes_{\mathcal{E}} W^{1}, V^{2}=\mathcal{V} \otimes_{\mathcal{E}} W^{2}$ and $V=\mathcal{V} \otimes_{\mathcal{E}} W$ resepectively. Then

$$
\begin{aligned}
V_{d} & \simeq \oplus_{a b^{-1}=d} P\left(a, b^{-1}\right)^{*} \otimes W_{a, b} \simeq \oplus_{a b^{-1}=d} P\left(a, b^{-1}\right)^{*} \otimes\left(W^{1} \otimes W^{2}\right)_{a, b} \\
& \simeq \oplus_{a b^{-1}=d} \oplus_{a^{\prime} a^{\prime \prime}=a} P\left(a, b^{-1}\right)^{*} \otimes P\left(a^{\prime}, a^{\prime \prime}\right)^{*} \otimes W_{a^{\prime}, e}^{1} \otimes W_{a^{\prime \prime}, b}^{2} \\
& \simeq \oplus_{d^{\prime} d^{\prime \prime}=d} \oplus_{a^{\prime \prime} b^{-1}=d^{\prime \prime}} P\left(d^{\prime}, d^{\prime \prime}\right)^{*} \otimes P\left(a^{\prime \prime}, b^{-1}\right)^{*} \otimes W_{d^{\prime}, e}^{1} \otimes W_{a^{\prime \prime}, b}^{2} \\
& \simeq \oplus_{d^{\prime} d^{\prime \prime}=d} P\left(d^{\prime}, d^{\prime \prime}\right)^{*} \otimes V_{d^{\prime}}^{1} \otimes V_{d^{\prime \prime}}^{2} \simeq\left(V^{1} \otimes V^{2}\right)_{d},
\end{aligned}
$$

so that $V \simeq V^{1} \otimes V^{2}$.

\subsection{Braiding on $\Delta-\Delta$ equivariant bundles}

We can use this Morita equivalence to understand the braiding on the representations of super-tube algebra $\mathcal{E}$. Thus the $\Delta-\Delta$ twisted equivariant bundles on $G \times G$ becomes a braided tensor category.

Suppose that $W^{1}, W^{2}$ are $\mathcal{E}$ modules. Then we form the fusion product module $W=W^{1} \otimes W^{2}$, and the corresponding $\mathcal{D}$ modules, $V^{1}=\mathcal{V} \otimes_{\mathcal{E}}$ $W^{1}, V^{2}=\mathcal{V} \otimes_{\mathcal{E}} W^{2}$ and $V=\mathcal{V} \otimes_{\mathcal{E}} W$ resepectively, so that $W^{1} \otimes W^{2} \simeq$ $\mathcal{V} \otimes_{\mathcal{D}} V^{1} \otimes V^{2}$. The braiding on $\operatorname{Rep}\left(\mathcal{E}^{\omega}(\mathrm{G})\right)$ is then simply: $\varepsilon\left(W^{1}, W^{2}\right)$ from $W^{1} \otimes W^{2} \rightarrow W^{2} \otimes W^{1}$ given by

$$
\varepsilon\left(W^{1}, W^{2}\right)=1_{\mathcal{V}} \otimes_{\mathcal{D}} \varepsilon\left(V^{1}, V^{2}\right): \mathcal{V} \otimes_{\mathcal{D}} V^{1} \otimes V^{2} \rightarrow \mathcal{V} \otimes_{\mathcal{D}} V^{2} \otimes V^{1}
$$

To be more explicit in terms of twisted vector bundles, we first have: 


$$
\begin{aligned}
\left(V^{1} \otimes V^{2}\right)_{d} & \simeq \oplus_{d^{\prime} d^{\prime \prime}=d} P\left(d^{\prime}, d^{\prime \prime}\right)^{*} \otimes V_{d^{\prime}}^{1} \otimes V_{d^{\prime \prime}}^{2} \\
& \simeq \oplus_{d^{\prime} d^{\prime \prime}=d} \oplus_{a^{\prime \prime} b^{-1}=d^{\prime \prime}} P\left(d^{\prime}, d^{\prime \prime}\right)^{*} \otimes P\left(a^{\prime \prime}, b^{-1}\right)^{*} \otimes W_{d^{\prime}, e}^{1} \otimes W_{a^{\prime \prime}, b}^{2},
\end{aligned}
$$

If $v^{1} \in V^{1}, v^{2} \in V^{2}$, then according to Eq. (27), we have

$$
\varepsilon\left(V^{1}, V^{2}\right)\left[v_{d^{\prime}}^{1} \otimes v_{d^{\prime \prime}}^{2}\right]=d^{\prime} \cdot v_{d^{\prime \prime}}^{2} \otimes v_{d^{\prime}}^{1} \quad \in V_{d^{\prime} d^{\prime \prime} d^{\prime-1}}^{2} \otimes V_{d^{\prime}}^{1}
$$

If $w^{1} \in W^{1}, w^{2} \in W^{2}$, we define $v^{1} \in V^{1}, v^{2} \in V^{2}$ by

$$
\begin{gathered}
v_{d^{\prime}}^{1}=v\left(\left(d^{\prime}, e\right), e\right) \otimes w_{d^{\prime}, e}^{1}, \\
v_{d^{\prime \prime}}^{2}=v\left(\left(d^{\prime \prime}, e\right), e\right) \otimes w_{d^{\prime \prime}, e}^{2} .
\end{gathered}
$$

Writing $v=v^{2}, w=w^{2}$, we compute:

$$
\begin{aligned}
d^{\prime} v_{d^{\prime \prime}} & =c\left(d^{\prime \prime}, d^{\prime}\right) v\left(\left(d^{\prime \prime}, e\right), e\right) \otimes w_{d^{\prime \prime}, e} \\
& =v\left(\left(d^{\prime} d^{\prime \prime} d^{\prime-1}, e\right), e\right) t\left(d^{\prime},\left(d^{\prime \prime}, e\right), d^{\prime}\right) w_{d^{\prime \prime}, e} \\
& =v\left(\left(d^{\prime} d^{\prime \prime} d^{\prime-1}, e\right), e\right) t\left(e, d^{\prime}\left(d^{\prime \prime}, e\right), d^{\prime}\right) t\left(d^{\prime},\left(d^{\prime \prime}, e\right), e\right) w_{d^{\prime \prime}, e} \\
& =v\left(\left(d^{\prime} d^{\prime \prime} d^{\prime-1}, e\right), e\right) \omega\left(d^{\prime} d^{\prime \prime}, d^{\prime-1}, d^{\prime}\right) \bar{\omega}\left(d^{\prime}, d^{\prime-1}, d^{\prime}\right)\left(d^{\prime} w_{d^{\prime \prime}, e}^{2}\right) d^{\prime-1} .
\end{aligned}
$$

Untangling this, we get that

$$
\begin{gathered}
\varepsilon\left(W^{1}, W^{2}\right)\left[w_{d^{\prime}, e}^{1} \otimes w_{d^{\prime \prime}, e}^{2}\right]= \\
\omega\left(d^{\prime} d^{\prime \prime}, d^{\prime-1}, d^{\prime}\right) \bar{\omega}\left(d^{\prime}, d^{\prime-1}, d^{\prime}\right)\left(d^{\prime} w_{d^{\prime \prime}}^{2}\right) d^{\prime-1} \otimes w_{d^{\prime}, e}^{1} \in W_{d^{\prime} d^{\prime \prime} d^{\prime-1}, e}^{2} \otimes W_{d^{\prime}, e}^{1} .
\end{gathered}
$$

By equivariance, this is enough to compute the brading on anything. For example, we have:

$$
\begin{aligned}
& \varepsilon\left(W^{1}, W^{2}\right)\left[w_{e, b}^{1} \otimes w_{a, e}^{2}\right] \\
& =\omega\left(b, b^{-1}, b\right) \varepsilon\left(W^{1}, W^{2}\right)\left[b\left(b^{-1} w_{e, b}^{1}\right) \otimes w_{a, e}^{2}\right] \\
& =\omega\left(b, b^{-1}, b\right) \bar{\omega}\left(b, b^{-1}, a\right) \varepsilon\left(W^{1}, W^{2}\right)\left[b\left(b^{-1} w_{e, b}^{1} \otimes w_{a, e}^{2}\right)\right] \\
& =\omega\left(b, b^{-1}, b\right) \bar{\omega}\left(b, b^{-1}, a\right) b \varepsilon\left(W^{1}, W^{2}\right)\left[b^{-1} w_{e, b}^{1} \otimes w_{a, e}^{2}\right] \\
& =\omega\left(b, b^{-1}, b\right) \bar{\omega}\left(b, b^{-1}, a\right) \omega\left(b^{-1} a, b, b^{-1}\right) \bar{\omega}\left(b^{-1}, b, b^{-1}\right) b \cdot\left[\left(b^{-1} w_{a, e}^{2}\right) b \otimes b^{-1} w_{e, b}^{1}\right] \\
& =\omega\left(b, b^{-1}, b\right) \bar{\omega}\left(b, b^{-1}, a\right) \bar{\omega}\left(b^{-1}, b, b^{-1}\right) b \cdot\left[b^{-1} w_{a, e}^{2} \otimes b\left(b^{-1} w_{e, b}^{1}\right)\right] \\
& =\bar{\omega}\left(b, b^{-1}, a\right) \bar{\omega}\left(b^{-1}, b, b^{-1}\right) b \cdot\left[b^{-1} w_{a, e}^{2} \otimes w_{e, b}^{1}\right] \\
& =\bar{\omega}\left(b, b^{-1}, a\right)\left[b \cdot\left(b^{-1} w_{a, e}^{2}\right) \otimes w_{e, b}^{1}\right] \\
& =w_{a, e}^{2} \otimes w_{e, b}^{1} .
\end{aligned}
$$

We record this as:

$$
\varepsilon\left(W^{1}, W^{2}\right)\left[w_{e, b}^{1} \otimes w_{a, e}^{2}\right]=w_{a, e}^{2} \otimes w_{e, b}^{1} .
$$

In the case of trivial twisting $\omega=1$, we have

$$
\varepsilon\left(W^{1}, W^{2}\right)\left[w_{a_{1}, b_{1}}^{1} \otimes w_{a_{2}, b_{2}}^{2}\right]=a_{1} w_{a_{2}, b_{2}}^{2} b_{2}^{-1} \otimes a_{1}^{-1} w_{a_{1}, b_{1}}^{1} b_{2} .
$$

Taking inverses, $\varepsilon\left(W^{1}, W^{2}\right)^{-1}: W^{2} \otimes W^{1} \rightarrow W^{1} \otimes W^{2}$ will then be determined as:

$$
\varepsilon(V, W)^{-1}\left[w_{a_{2}, b_{2}}^{2} \otimes w_{a_{1}, b_{1}}^{1}\right]=b_{2} w_{a_{1}, b_{1}}^{1} a_{1}^{-1} \otimes b_{2}^{-1} w_{a_{2}, b_{2}}^{2} a_{1} .
$$




\section{Twisted equivariant bundles over finite groups}

Let $\Gamma$ be a finite group, $\alpha$ a 3-cocycle in $Z^{3}(\Gamma, \mathbb{T})$, with $H$ and $K$ subgroups of $\Gamma$, and $\psi$ and $\psi^{\prime}$ are 2-cocycles in $Z^{2}(H, \mathbb{T}), Z^{2}(K, \mathbb{T})$ respectively. We consider a $H$ - $K$ bundle $V$ twisted by $\alpha$ with base space $\Gamma$, where $H$ and $K$ act on the fibres on the left and right with multipliers $\psi$ and $\psi^{\prime}$ respectively, satisfying the following consistency relations:

$$
\begin{array}{r}
\left(h_{1} h_{2}\right) w=\bar{\alpha}\left(h_{1}, h_{2}, g\right) \psi\left(h_{1}, h_{2}\right)\left(h_{1}\left(h_{2} w\right)\right), \\
w\left(k_{1} k_{2}\right)=\alpha\left(g, k_{1}, k_{2}\right)^{-1} \psi^{\prime}\left(k_{1}, k_{2}\right)\left(\left(w k_{1}\right)\left(k_{2}\right),\right. \\
h(w k)=\alpha(h, g, k)(h w) k,
\end{array}
$$

where $h_{1}, h_{2}, h \in H, k_{1}, k_{2}, k \in K$, and $w=w_{g} \in V_{g}$, the fibre over $g \in$ $\Gamma$. Here $h w, w k$ lie in the fibres over $h g$, and $g k$ respectively, etc. We let ${ }^{\alpha} \operatorname{Bun}^{H-K}(\Gamma)$ denote such twisted bundles.

The equivalence classes generate the equivariant twisted $K$-group ${ }^{\alpha} K_{0}^{H \times K}(\Gamma)$. If $V$ is an $H$ - $K$ bundle, we can naturally associate the conjugate $K-H$ bundle $V^{*}$. If $L$ is another subgroup of $\Gamma$, we can naturally form from an $H-K$ bundle $V$ and a $K-L$ bundle $W$ a $H-L$ bundle $V \otimes_{K} W$ :

$$
{ }^{\alpha} \operatorname{Bun}^{H \times K}(\Gamma) \times{ }^{\alpha} \operatorname{Bun}^{K \times L}(\Gamma) \rightarrow{ }^{\alpha} \operatorname{Bun}^{H \times L}(\Gamma)
$$

and hence a product on K-theory:

$$
{ }^{\alpha} K_{0}^{H \times K}(\Gamma) \times{ }^{\alpha} K_{0}^{K \times L}(\Gamma) \rightarrow{ }^{\alpha} K_{0}^{H \times L}(\Gamma) .
$$

We divide the tensor product $V \otimes W$ over $\Gamma \times \Gamma$ by the relation:

$$
v_{a} k \otimes w_{b}=\alpha(a, k, b) v_{a} \otimes k w_{b}
$$

and then push forward under the product map $\Gamma \times \Gamma \rightarrow \Gamma$ to obtain $V \otimes_{K} W$, a bundle over $\Gamma$ where $\left(V \otimes_{K} W\right)_{g}=\oplus_{a b=g} V_{a} \otimes W_{b}$. Then $V \otimes_{K} W$ becomes a $H-K \alpha$ twisted bundle ${ }_{H} V \otimes_{K} W_{L}$ under the natural actions:

$$
\begin{array}{r}
h\left(v_{a} \otimes w_{b}\right)=\alpha(h, a, b) h v_{a} \otimes w_{b} \\
\left(v_{a} \otimes w_{b}\right) l=\bar{\alpha}(a, b, l) v_{a} \otimes w_{b} l
\end{array}
$$

Now if $V$ is a bundle, we let $s(V)$ denote its support $\left\{g \in \Gamma: V_{g} \neq 0\right\}$. For an irreducible bundle, the support $s(V)$ is a single double coset $H g K$. To compute the equivariant K-group ${ }^{\alpha} K_{0}^{H \times K}(\Gamma)$, we first take representatives for the double cosets $H \backslash \Gamma / K$. Then for each double coset $H g K$ we consider the stabilizer subgroup $H \times{ }_{g} K=\{(h, k) \in H \times K: h g=g k\}$ which is isomorphic to $H \cap{ }^{g} K$ and $H^{g} \cap K$, where ${ }^{g} K=g K g^{-1}, H^{g}=g^{-1} H g$, under the projections $\left(h, g^{-1} h g\right)=\left(g k g^{-1}, k\right) \rightarrow$ to $h$ and $k$ respectively as $h$ determines $k$ and vice versa. Then $(h, k): w_{g} \rightarrow h\left(w_{g} k\right)$ gives a projective representation of $H \times{ }_{g} K$ on $V_{g}$, with multiplier or 2-cocycle 


$$
\begin{gathered}
\alpha^{g}\left(h, h^{\prime}\right):=\psi_{1}\left(h, h^{\prime}\right) \psi_{2}\left(g^{-1} h^{\prime^{-1}} g, g^{-1} h^{-1} g\right) \bar{\alpha}\left(h h^{\prime} g, g^{-1} h^{\prime-1} g, g^{-1} h^{-1} g\right) \\
\times \alpha\left(h, h^{\prime}, g\right) \alpha\left(h, h^{\prime} g, g^{-1} h^{\prime-1} g\right) .
\end{gathered}
$$

Then the irreducible bundles are labelled by a coset and an irreducible projective representation of the stabilizer.

Suppose $G$ is a finite group and let $\Gamma=G \times G$, and $\Delta(G)=\{(g, g)$ : $g \in G\}$ denote the diagonal subgroup which we denote simply by $\Delta$ when there is no confusion. If $\omega$ is a 3-cocycle in $Z^{3}(G, \mathbb{T})$, we define the 3-cocycle $\alpha=\pi_{1}^{*} \omega-\pi_{2}^{*} \omega$ on $G \times G$ if $\pi_{1}, \pi_{2}$ are the projections of $G \times G$ on the first and second factors respectively.

The Verlinde algebra ${ }_{N} \mathcal{X}_{N}$ for the quantum double of $G$ is then the space of $\Delta$ - $\Delta$ bundles or the equivariant $K$-group $K_{0}^{\Delta \times \Delta}(G \times G)$. Since

$$
\Delta(G)(g, h) \Delta(G)=\Delta(G)\left(g h^{-1}, 1\right) \Delta(G)
$$

for any $g, h \in G$, it is the case that every double coset $\Delta(G)(g, h) \Delta(G)$ gives rise to a conjugacy class $C_{g h^{-1}}$ of $\Delta(G)$. Moreover, the stabilizer of $(g, h)$ equals the centraliser of $g h^{-1}$, i.e.

$$
(g, h) \Delta(G)\left(g^{-1}, h^{-1}\right) \cap \Delta(G)=\left\{x \in G: x g h^{-1}=g h^{-1} x\right\} .
$$

Consequently, the primary fields or irreducible bundles are given by pairs $(a, \chi)$ where $a$ are representatives of conjugacy classes of $G$ and $\chi$ are irreducible representations of the centraliser $C_{G}(a)$ of $a \in G$.

There are a number of special cases of particular interest. One is when $H=\Delta$, and $\psi=1$. The double cosets $\Delta \backslash \Gamma / K$ are labelled by

$$
\Delta(G)(g, h) K=\Delta(G)\left(g h^{-1}, 1\right) K
$$

i.e. of the form $\Delta(x, 1) K$ where $x$ in $G$ is defined up to an action of $(h, k) \in$ $K \times K$ by conjugation $x \rightarrow h x k^{-1}$. For each such $x$, we identify the stabiliser subgroup $\Delta \times_{(x, 1)} K$ with the subgroup $K^{x}=\left\{\left(h_{1}, h_{2}\right) \in K: h_{1} x h_{2}^{-1}=x\right\}$ of $K$. Again, since $h_{2}$ is determined by $h_{1}$ the group $K^{x}$ can be understood as a subgroup of $G$ through projecting $K \subset G \times G$ to the first component. The multiplier $\omega^{(x, 1)}$ of the subgroup $\Delta \times_{(x, 1)} K$ is then regarded as a multiplier on $K^{x}$.

\subsection{The Frobenius algebra}

For the remaining exposition, we take for simplicity the case of trivial twist or level zero $\omega=1$ in $Z^{3}(G, \mathbb{T})$, for a finite group $G$. The quantum double of the group $G$ is idenified with the inclusion $N=M_{0} \rtimes \Delta(G) \subset M_{0} \rtimes(G \times G)=$ $M=M_{\Delta}$ where $\Delta(G)=\{(g, g): g \in G\}$ denotes the diagonal subgroup of $G \times G$. The $N-N$ system is described by $\operatorname{Bun}^{\Delta-\Delta}(\Gamma)$, if $\Gamma=G \times G$ [KY, KMY, EP2]. 
For $H$ a subgroup of $G \times G$, we define an irreducible element $\iota$, a bundle in $\operatorname{Bun}^{H-\Delta}(\Gamma)$, using the trivial representation on the trivial double coset:

$$
\iota=\iota_{H}=[H \Delta, 0]
$$

and similarly,

$$
\bar{\iota}=\bar{\iota}_{H}=[\Delta H, 0]
$$

in $B u n^{\Delta-H}(\Gamma)$. Again for simplicity, we take $\psi=1$ in $Z^{2}(H, \mathbb{T})$, We compute the products using $[\mathrm{KMY}]$ :

$$
\begin{aligned}
& \theta=\overline{\iota \iota}=\sum_{h \in \Delta \cap H \backslash H / \Delta \cap H}\left[\Delta h \Delta, \operatorname{Ind}(0)_{\Delta^{h} \cap H \cap \Delta}^{\Delta^{h} \cap \Delta}\right], \\
& \gamma=\iota \bar{\iota}=\sum_{k \in \Delta \cap H \backslash \Delta / \Delta \cap H}\left[H k H, \operatorname{Ind}(0)_{H^{k} \cap H \cap \Delta}^{H^{k} \cap H}\right],
\end{aligned}
$$

in $B u n^{\Delta-\Delta}(\Gamma), B u n^{H-H}(\Gamma)$ respectively. The former yields a Frobenius algebra $\Theta=\Theta_{H}$, or Q-system in the braided tensor category $\operatorname{Bun}^{\Delta-\Delta}(\Gamma)$. Since this is identified with the $N-N$ system, we thus have a subfactor $N \subset M_{H}$. Thus if we have two such subgroups $H_{a}$ and $H_{b}$ of $\Gamma$, then the corresponding $M_{a}-M_{b}$ system is identified with $\operatorname{Bun}^{H_{a}-H_{b}}(\Gamma)$ ( the irreducible components of $\left\{\iota_{a} \lambda \bar{\iota}_{b}: \lambda\right\}$. In particular, we identify the $N-M_{H}$ sectors with $B u n^{\Delta-H}(\Gamma)$ and the $M_{H}-M_{H}$ system with $B u n^{H-H}(\Gamma)$.

In particular, for the special cases:

$$
\begin{gathered}
\Delta \subset H: \overline{\iota \iota}=\sum_{h \in \Delta \backslash H / \Delta}[\Delta h \Delta, 0]=[H, 0], \\
H \subset \Delta: \overline{\iota \iota}=\left[\Delta, \operatorname{Ind}(0)_{H}^{\Delta}\right] .
\end{gathered}
$$

\section{$3.2 \alpha$-induction and modular invariants}

We can use the Frobenius algebra $\Theta=\Theta_{H}$ or Q-system for each $H$ to define $\alpha$-induction from $\operatorname{Bun}^{\Delta-\Delta}(\Gamma)$ to $\operatorname{Bun}^{H-H}(\Gamma)$ and hence construct a modular invariant.

We identify $\operatorname{Bun}^{H-\Delta}(\Gamma)$, with left $\Theta$ modules $B u n^{\Delta-H}(\Gamma)$ with right $\Theta$ modules and $\operatorname{Bun}^{H-H}(\Gamma)$, with $\Theta-\Theta$ bimodules. More generally, if $\Theta_{a}=$ $\Theta_{H_{a}}$, and $\Theta_{b}=\theta_{H_{b}}$, we identify following [EP2] $B n^{H_{a}-H_{b}}(\Gamma)$ with $\Theta_{a^{-}}$ $\Theta_{b}$ bimodules. Recall that a $\Theta_{a}-\Theta_{b}$ bimodule [O1, EP2] is an element $M$ of Bun $^{\Delta-\Delta}(\Gamma)$ with morphisms from $\theta_{a} \otimes_{G} M$ and $M \otimes_{G} \theta_{b}$ into $M$ satisfying natural compatibility conditions.

Every irreducible $\beta$ in $\operatorname{Bun}^{H_{a}-H_{b}}(\Gamma)$ arises from the decomposition $\iota_{a} \lambda \bar{\iota}_{b}$ with $\lambda$ in $\operatorname{Bun}^{\Delta-\Delta}(\Gamma)$. Define now $\Phi: \operatorname{Bun}^{H_{a}-H_{b}}(\Gamma) \rightarrow \Theta_{a}-\Theta_{b}$-bimodules by $\Phi(\beta)=\bar{\iota}_{a} \beta \iota_{b}$ for $\beta \in \operatorname{Bun}^{H_{a}-H_{b}}(\Gamma)$. In particular, $\Phi\left(\iota_{a} \lambda \bar{\iota}_{b}\right)=\theta_{a} \lambda \theta_{b}$. If $\beta, \beta^{\prime} \in \operatorname{Bun}^{H_{1}-H_{2}}(\Gamma)$, then we map an intertwiner $t \in \operatorname{Hom}\left(\beta, \beta^{\prime}\right)$ to $\Phi(t)=\bar{\iota}_{a} t \iota_{b} \in \operatorname{Hom}\left(\bar{\iota}_{a} \beta \iota_{b}, \bar{\iota}_{a} \beta^{\prime} \iota_{b}\right)=\operatorname{Hom}\left(\Phi(\beta), \Phi\left(\beta^{\prime}\right)\right.$. Then $\Phi$ is a $\Theta_{a}-\Theta_{b}$ 
morphism. We note that $\Phi$ is injective. Suppose that $\bar{\iota}_{a} \beta \iota_{b} \simeq \bar{\iota}_{a} \beta^{\prime} \iota_{b}$ as $\Theta_{a^{-}}$ $\Theta_{b}$ bimodules. Then $t=\mathbf{1}_{\bar{\iota}_{a}} \otimes t^{\prime \prime} \otimes \mathbf{1}_{\iota_{b}}$ with $t^{\prime \prime} \in \operatorname{Hom}\left(\beta, \beta^{\prime}\right)$. If $t$ is an isomorphism so is $t^{\prime \prime}$, therefore $\beta \simeq \beta^{\prime}$. That $\Phi$ is surjective can be seen by counting dimension (cf. proof of Lemma 3.1 in [EP2].

In this formulation, $\alpha$-induction looks as follows. Take a bundle $V$ in $\operatorname{Bun}^{\Delta-\Delta}(\Gamma)$, and form the bundle $V \otimes_{G} \theta$ again in ${ }^{\omega} \operatorname{Bun}^{\Delta-\Delta}(\Gamma)$. The latter determines a $\Theta-\Theta$ bimodule, since $\theta$ itself is a $\Theta-\Theta$ bimodule. Consequently, $V \otimes_{G} \theta$ becomes a $\Theta-\Theta$ bimodule using the natural action of $\theta$ on the right on $\theta$, and the braiding $\varepsilon^{ \pm}(V, \theta)$ to hit $\theta$ in $V \otimes_{G} \theta$ on the left. We can then identify these induced bimodules $\alpha_{V}^{ \pm}$with elements of $B u n^{H-H}(\Gamma)$ using the previous paragraph.

In the special case when $\Delta \subset H \subset \Gamma$, we can also view this construction as follows. For a bundle $V$ in $\operatorname{Bun}^{\Delta-\Delta}(\Gamma)$ we form the product $\Delta-\Delta$ bundle $V \otimes_{\Delta} \theta$. This can be considered as a $H-H$ bundle since $\theta$ can be viewed as a $H$ - $H$ bundle, and so $V \otimes_{\Delta} \theta$ has a natural right $H$ action on $\theta$, and using the braiding to identify $V \otimes_{\Delta} \theta$ with $\theta \otimes_{\Delta} V$, which has a natural left $H$ action. Since we can use the braiding $\varepsilon^{+}=\varepsilon(V, \theta)$ or its adjoint $\varepsilon^{-}=\varepsilon(V, \theta)^{*}$, we can form two inductions $\alpha^{ \pm}$in this way. Note that the relation $\iota \otimes_{G} V \simeq \alpha_{V}^{ \pm} \otimes_{H} \iota_{G}$ as $H$ - $G$ bundles since $\iota \otimes_{G} V \simeq\left(V \otimes_{G} \bar{\iota} \iota\right) \otimes_{H} \iota$, as $\theta \otimes_{H} \iota \simeq \iota$ as $H$-G bundles.

Any such subgroup $\Delta \subset H \subset \Gamma$, is of the form $H=\Delta(1, N)=\Delta(N, 1)$, where $N$ is a normal subgroup of $G$ (or $\Delta$ ). Indeed $N=p(H)$, where $p$ is the projection $\left(g_{1}, g_{2}\right) \rightarrow g_{1} g_{2}^{-1}$ from $\Gamma$ to $G$. Clearly any such subgroup $H$ is invariant under the flip $\sigma$ on $\Gamma$. We will see in the examples of Sect. 4 that the neutral system can be identified with the non-degenerately braided system, $\operatorname{Bun}^{\Delta(N)-\Delta(N)}(N \times N)$, i.e. the quantum double of $N$.

We compute explicitly the induced bundle $\alpha_{V}^{ \pm}$when $V=[\Delta a \Delta, \chi]$ is an irreducible $\Delta-\Delta$ bundle, with $a \in \Gamma$ and a representation $\pi$ of the stabiliser $\Delta_{a}=\Delta \cap^{a} \Delta$ with character $\chi$. We have

$$
\begin{aligned}
h^{+}\left(v_{a_{1} b_{1}} \otimes e_{a_{2} b_{2}}\right) & =\varepsilon(V, \theta)^{-1}\left[h\left(\varepsilon(V, \theta)\left(a_{1}\left(e_{a_{2}, b_{2}} b_{2}^{-1}\right) \otimes\left(a_{1}^{-1} v_{a_{1}, b_{1}}\right) b_{2}\right)\right]\right. \\
& =\varepsilon(V, \theta)^{-1}\left[h \cdot\left(a_{1}\left(e_{a_{2}, b_{2}} b_{2}^{-1}\right) \otimes\left(a_{1}^{-1} v_{a_{1}, b_{1}}\right) b_{2}\right)\right] \\
& \left.=\varepsilon(V, \theta)^{-1}\left[h a_{1}\left(e_{a_{2}, b_{2}} b_{2}^{-1}\right)\right) \otimes\left(a_{1}^{-1} v_{a_{1}, b_{1}}\right) b_{2}\right] \\
& =h_{1} a_{1}\left[a_{1}^{-1} v_{a_{1} b_{1}} b_{2}\right] b_{2}^{-1} \otimes\left(h_{1} a_{1}\right)^{-1}\left[h a_{1}\left(e_{a_{2}, b_{2}} b_{2}^{-1}\right)\right] b_{2} \\
& =h_{1} v_{a_{1} b_{1}}\left(a d ( a _ { 1 } ^ { - 1 } ) ( h _ { 1 } ) \otimes \left(a d\left(a_{1}^{-1}(h)\right)\left(e_{a_{2}, b_{2}}\right) .\right.\right.
\end{aligned}
$$

We arrive at:

$$
\alpha_{[\Delta a \Delta, \pi]}^{ \pm}=\left[H a H, \operatorname{Ind}_{\Delta \cap a \Delta \mathrm{a}^{-1}}^{\Delta \cap \mathrm{aHa}^{-1}}(\pi) \pi^{ \pm}\right]=\left[\mathrm{HaH}, \pi_{ \pm}\right]
$$

for $\pi \in \operatorname{Rep}\left[\Delta \cap \mathrm{a} \Delta \mathrm{a}^{-1}\right]$ where $\pi^{ \pm}:\left(h_{+}, h_{-}\right) \rightarrow\left(h_{+}, h_{+}\right)$or $\left(\mathrm{h}_{-}, \mathrm{h}_{-}\right)$takes $H \cap a H a^{-1} \rightarrow \Delta \cap a H a^{-1}$.

Let us examine this case $\Delta \subset H \subset \Gamma$ when $G$ is abelian. Then

$$
\pi_{+}\left(h_{1}, h_{2}\right)=\pi\left(h_{1}\right), \pi_{-}\left(h_{1}, h_{2}\right)=\pi\left(h_{2}\right) .
$$


This means that:

$$
\begin{aligned}
\alpha_{[a \Delta, \chi]}^{+} & =\left[a H,\left.(\chi \times 1)\right|_{H}\right], \\
\alpha_{[a \Delta, \chi]}^{-} & =\left[a H,\left.(1 \times \chi)\right|_{H}\right] .
\end{aligned}
$$

Now we can write $H=\Delta \times N$, as sets using the identification $(a, b) \rightarrow$ $\left(a, a^{-1} b\right)=(\delta, n)$, so that $\left.(\chi \times 1)\right|_{H}(a, b)=\chi(\delta),\left.(1 \times \psi)\right|_{H}(a, b)=\psi(\delta) \psi(n)$. Then

$$
\begin{gathered}
\left\langle\left.(\chi \times 1)\right|_{H},\left.(\psi \times 1)\right|_{H}\right\rangle=\langle\chi, \psi\rangle, \\
\left\langle\left.(\chi \times 1)\right|_{H},\left.(1 \times \psi)\right|_{H}\right\rangle=\langle\chi, \psi\rangle\left\langle\left.\psi\right|_{N}, 1\right\rangle .
\end{gathered}
$$

Consequently, $\left\langle\alpha_{[a \Delta, \chi]}^{+}, \alpha_{[b \Delta, \psi]}^{+}\right\rangle=1$, if $a b^{-1} \in H, \chi=\psi$, and 0 otherwise. Moreover, the modular invariant mass matrix is given as $\left\langle\alpha_{[a \Delta, \chi]}^{+}, \alpha_{[b \Delta, \psi]}^{-}\right\rangle=1$ if $a b^{-1} \in H, \chi=\psi$ and $\left.\chi\right|_{N}=1$, and 0 otherwise.

\section{Examples}

For a cyclic groups $\mathbb{Z}_{d}$, the primary fields are parametrized by pairs $(m, n)$ for $m, n \in \mathbb{Z}_{d}$ (the first factor labels the double cosets and the second the stabilisers) whose conjugate is $(-m,-n)$, and the $S$ and $T$ matrices:

$$
\begin{aligned}
S_{(m, n),\left(m^{\prime}, n^{\prime}\right)} & =d^{-1} \exp \left[-2 \pi \sqrt{-1}\left(n m^{\prime}+m n^{\prime}\right) / d\right], \\
T_{(m, n),(m, n)} & =\exp [(2 \pi \sqrt{-1} n m / d)] .
\end{aligned}
$$

When $d=p$ is a prime number, the complete list of all modular invariants is described in [CGP, EP4]. There are four non-permutation modular invariants: $\mathcal{Z}_{4}=x x^{*}, \mathcal{Z}_{7}=x y^{*}, \mathcal{Z}_{8}=y x^{*}$, and $\mathcal{Z}_{5}=y y^{*}$ where $x=\sum_{i=0}^{p-1} \chi_{i 0}$ and $y=\sum_{j=0}^{p-1} \chi_{0 j}$. For any prime $p>2$, there will be four permutation modular invariants: $\mathcal{Z}_{1}=\sum_{i, j=0}^{p-1} \chi_{i j} \chi_{i j}^{*}, \mathcal{Z}_{2}=\sum_{i, j=0}^{p-1} \chi_{i j} \chi_{j i}^{*}, \mathcal{Z}_{3}=\sum_{i, j=0}^{p-1} \chi_{i j} \chi_{-j,-i}^{*}$ and $\mathcal{Z}_{6}=\sum_{i, j=0}^{p-1} \chi_{i j} \chi_{-i,-j}^{*}$ the charge conjugation. When $p=2, \mathcal{Z}_{6}=\mathcal{Z}_{1}$ and $\mathcal{Z}_{3}=\mathcal{Z}_{2}$ and we have six distinct modular invariants. When $p=3$, there are precisely eight distinct modular invariants. In the cases $p=2,3$, these exhaust all the modular invariants.

\section{1 $G=\mathbb{Z}_{2}$}

Here there are six distinct modular invariants, four of which are symmetric. All can be realised from subfactors or from module categories. The following are the 5 subgroups of $\mathbb{Z}_{2} \times \mathbb{Z}_{2}$ : 


$$
\begin{aligned}
& H_{1}=\{(0,0)\}, \\
& H_{3}=\{(0,0),(1,1)\}=\Delta\left(\mathbb{Z}_{2}\right), \\
& H_{4}=\mathbb{Z}_{2} \times\{0\}, \\
& H_{5}=\{0\} \times \mathbb{Z}_{2}, \\
& H_{6}=\mathbb{Z}_{2} \times \mathbb{Z}_{2} .
\end{aligned}
$$

In this case the 2-cohomology groups $H^{2}(H, \mathbb{T})$ are all trivial except for $H_{6}$ when it is $\mathbb{Z}_{2}$ and only $H_{1}, H_{3}$ and $H_{6}$ give rise to type I modular invariants or commutative Q-systems.

(i) Example: $H_{3}$ and $H_{6}$.

The subgroups $H_{3}$ and $H_{6}$ contain $\Delta$ with $N=0$, and $N=G=\mathbb{Z}_{2}$ respectively. We see that the corresponding modular invariants are $\mathcal{Z}=1$, and $X_{c}=x x^{*}=\mathcal{Z}_{4}$.

(ii) Example: $H_{1}$.

The subgroup $H_{1} \subset \Delta$. To understand $\alpha$-induction we first take $V=$ $[x, \psi] \rightarrow \iota[x, \psi] \bar{l}$. Now Bun ${ }^{H_{1}-H_{1}}(\Gamma) \simeq \operatorname{Bun}(\Gamma)$, and $\alpha^{ \pm}$-induction takes $[x, \psi] \rightarrow[(x, 0)],[(0, x)]$ respectively. Consequently, the corresponding modular invariant is $\mathcal{Z}_{5}=y y^{*}=X_{s}$.

(iii) Example: $\mathrm{H}_{4}$ and $H_{5}$.

There are two further non symmetric groups $H_{4}=\mathbb{Z}_{2} \times\{0\}$, and $H_{5}=$ $\{0\} \times \mathbb{Z}_{2}$. Writing $H=H_{4}$, there are two double cosets $H(0,0)=\mathbb{Z}_{2} \times\{0\}$ and $H(0,1)=\mathbb{Z}_{2} \times\{1\}$, both with stabilisers $H$, so that there are four irreducible objects in $\mathrm{Bun}^{\mathrm{H}_{4}-H_{4}}(\Gamma)$ which we write as $[[i, j]]$, where $i=0,1$ represents the double coset $H(0, i)$, and $j$ is a character of $H$. The double cosets decompose as

$$
\begin{aligned}
& H \Delta(0,0) \Delta H=H \Delta=H(0,0)+H(0,1), \\
& H \Delta(0,1) \Delta H=H(0,0)+H(0,1) .
\end{aligned}
$$

Then $\alpha$-induction gives:

$$
\begin{array}{ll}
\alpha_{0,0}=[[0,0]], & \\
\alpha_{0,1}^{+}=[[0,0]], & \alpha_{0,1}^{-}=[[0,1]], \\
\alpha_{1,0}^{+}=[[1,0]], & \alpha_{1,0}^{-}=[[0,0]], \\
\alpha_{1,1}^{+}=[[1,0]], & \alpha_{1,1}^{-}=[[0,1]] .
\end{array}
$$

This gives the modular invariant $Q=x y^{*}$ and $Q^{t}=y x^{*}$.

(iv) Example: $H_{6}$ with nontrivial twist. This yields the permutation invariant $\mathcal{Z}_{2}=\mathcal{Z}_{3}$.

\section{$4.2 G=\mathbb{Z}_{3}$}

Here there are 8 distinct modular invariants. All can be realised from subfactors or module categories. The following are the 6 subgroups of $\mathbb{Z}_{3} \times \mathbb{Z}_{3}$ : 


$$
\begin{aligned}
& H_{1}=\{(0,0)\}, \\
& H_{2}=\{(0,0),(1,2),(2,1)\}, \\
& H_{3}=\{(0,0),(1,1),(2,2)\}=\Delta\left(\mathbb{Z}_{3}\right), \\
& H_{4}=\mathbb{Z}_{3} \times\{0\}, \\
& H_{5}=\{0\} \times \mathbb{Z}_{3}, \\
& H_{6}=\mathbb{Z}_{3} \times \mathbb{Z}_{3} . .
\end{aligned}
$$

In this case the cohomology $H^{2}(H, \mathbb{T})$ is nontrivial only for $H_{6}$ when it is $\mathbb{Z}_{3}$. Only $H_{1}, H_{2}, H_{3}$ and $H_{6}$ give rise to type I modular invariants or commutative Q-systems. As for $\mathbb{Z}_{2}$, the subgroups $H_{3}$ and $H_{6}$ give the modular invariants $\mathcal{Z}_{1}=1$ and $\mathcal{Z}_{4}=x x^{*}$ respectively and $H_{1}$ gives $\mathcal{Z}_{5}=y y^{*}$. The subgroups $H_{4}$ and $H_{5}$ give $\mathcal{Z}_{7}=x y^{*}$ and $\mathcal{Z}_{8}=y x^{*}$, whilst $H_{6}$ with its nontrivial twists from $H^{2}=\mathbb{Z}_{3}$ give $\mathcal{Z}_{2}$ and $\mathcal{Z}_{3}$. The remaining subgroup $H_{2}$ yields the conjugation invariant $\mathcal{Z}_{6}$.

\section{$4.3 G=S_{3}$}

There are 48 distinct modular invariants but only 28 can be realised from subfactors from module categories. There are 22 distinct non-conjugate subgroups of $S_{3} \times S_{3}$, which together with some non-trivial 2-cohomology is enough to produce all the 28 invariants. The following are the subgroups of $S_{3} \times S_{3}$ which give rise to type I modular invariants or commutative Q-systems:

$$
\begin{aligned}
& H_{1}=\{(1,1)\}, \\
& H_{4}=\Delta\left(\mathbb{Z}_{2}\right), \\
& H_{7}=\Delta\left(\mathbb{Z}_{3}\right), \\
& H_{8}=\mathbb{Z}_{2} \times \mathbb{Z}_{2}, \\
& H_{11}=\Delta\left(S_{3}\right), \\
& H_{14}=\mathbb{Z}_{3} \times \mathbb{Z}_{3}, \\
& H_{19}=K=\Delta\left(S_{3}\right) \cdot\left(1 \times \mathbb{Z}_{3}\right) \simeq\left(\mathbb{Z}_{3} \times \mathbb{Z}_{3}\right) \rtimes \mathbb{Z}_{2}, \\
& H_{20}=S_{3} \times S_{3} .
\end{aligned}
$$

The 2-cohomology $H^{2}(H, \mathbb{T})$ is non trivial only for $H_{8}, H_{14}, H_{19}, H_{20}$ in the above list as well as for $\mathbb{Z}_{2} \times S_{3}$ and $S_{3} \times \mathbb{Z}_{2}$.

The group $S_{3}$ is generated by $\sigma$ and $\tau$, with $\sigma^{3}=\tau^{2}=1, \tau \sigma \tau=\sigma^{2}$. There are three $\Delta$ - $\Delta$ double cosets: $\Delta(1,1) \Delta, \Delta(\sigma, 1) \Delta, \Delta(\tau, 1) \Delta$, with stabilisers isomorphic to $S_{3}, \mathbb{Z}_{3}, \mathbb{Z}_{2}$, respectively. Denote the correspoding irreducible representations by $\{1, \varepsilon, \pi\}$, (where $\varepsilon$ is the parity and $\pi$ the two dimensional representation), $\left\{1, \omega, \omega^{2}\right\},\{1, \epsilon\}$, and we denote in this order the corresponding irreducible bundles as $\{0,1,2,3,4,5,6,7\}$ as usual as in [CGP, EP2].

(i) Example: $H_{11}=\Delta\left(S_{3}\right), H_{19}=\left(\mathbb{Z}_{3} \times \mathbb{Z}_{3}\right) \rtimes \mathbb{Z}_{2}, H_{20}=S_{3} \times S_{3}$. 
The subgroups $H_{11}, H_{19}, H_{20}$ contain $\Delta$ and (in the case of the untwisted 2cohomology) have corresponding modular invariants $1, Z_{(33)}, Z_{55}$ respectively:

$$
\begin{aligned}
& Z_{(33)}=\left|\chi_{0}+\chi_{3}\right|^{2}+\left|\chi_{1}+\chi_{3}\right|^{2}+\left|\chi_{6}\right|^{2}+\left|\chi_{7}\right|^{2}, \\
& Z_{55}=\left|\chi_{0}+\chi_{3}+\chi_{6}\right|^{2} .
\end{aligned}
$$

Next consider the symmetric cases contained in $\Delta$, namely $H_{1}, H_{4}$ and $H_{7}$ (where $H^{2}$ always vanishes).

(ii) Example: $H_{1}=\{(1,1)\}$.

Take an irreducible bundle $[\Delta k \Delta, \psi]$ of $\operatorname{Bun}^{\Delta-\Delta}(\Gamma)$, where $\psi$ is an irreducible representation of the stabiliser $\Delta_{k}$ of the double coset $\Delta k \Delta$. Then, for the subgroup $H_{1}=0, \alpha^{ \pm}$-induction is again $[\Delta(g, 1) \Delta, \psi] \rightarrow \operatorname{dim}(\psi)[(\mathrm{g}, 1)]$ and $\operatorname{dim}(\psi)[(1, \mathrm{~g})]$ respectively, so that the corresponding modular invariant is

$$
\left\langle\alpha^{+}[\Delta(g, 1) \Delta, \psi], \alpha^{-}[\Delta(h, 1) \Delta, \chi]\right\rangle=\operatorname{dim}(\psi) \operatorname{dim}(\chi)\left[\delta_{\mathrm{g}, 1}, \delta_{\mathrm{h}, 1}\right],
$$

i.e. the mass matrix $Z_{22}$ :

$$
Z_{22}=\left|\chi_{0}+\chi_{1}+2 \chi_{2}\right|^{2}
$$

There are two further subgroups contained in $\Delta$, namely $H_{4}=\Delta\left(\mathbb{Z}_{2}\right)$ and $H_{7}=\Delta\left(\mathbb{Z}_{3}\right)$.

(iii) Example: $H_{4}=\Delta\left(\mathbb{Z}_{2}\right)$.

Consider first the case $H=H_{4}$, which has ten double cosets:

$$
\begin{aligned}
& H(1,1) H=H, H(1, \sigma) H, H(\sigma, 1) H, H(\sigma, \sigma) H, H\left(\sigma, \sigma^{2}\right) H, \\
& H(1, \sigma \tau) H, H(\sigma \tau, 1) H, H(\sigma \tau, \sigma) H, H\left(\sigma \tau, \sigma^{2}\right) H, H(1, \tau) H,
\end{aligned}
$$

with corresponding stabilisers $\mathbb{Z}_{2}$ for the first and last listed cosets and 1 for the remaining eight cosets, giving twelve irreducible bundles in $\mathrm{Bun}^{H-H}(\Gamma)$. We decompose as $H-H$ double cosets:

$$
\begin{aligned}
H \Delta(1,1) \Delta H= & H(1,1) H+H(\sigma, \sigma) H, \\
H \Delta(\sigma, 1) \Delta H= & H(\sigma, 1) H+H\left(\sigma, \sigma^{2}\right) H+H(1, \sigma) H, \\
H \Delta(\tau, 1) \Delta H= & H(\tau, 1) H+H(\sigma \tau, 1) H+H(1, \sigma \tau) H \\
& +H(\sigma, \sigma \tau) H+H\left(\sigma \tau, \sigma^{2}\right) H .
\end{aligned}
$$

Then $\alpha$-induction becomes:

$$
\begin{array}{ll}
\alpha_{1,1} & =[(1,1), 0)], \\
\alpha_{1, \epsilon}^{ \pm} & =[(1,1), 1)], \\
\alpha_{1, \pi}^{ \pm} & =[(1,1), 0)]+[(1,1), 1)], \\
\alpha_{(\sigma, 1), 1}^{ \pm} & =[(\sigma, 1), 0)], \quad[(1, \sigma), 0)], \\
\alpha_{(\sigma, 1), \omega}^{ \pm} & =[(\sigma, 1), 0)], \quad[(1, \sigma), 0)], \\
\alpha_{(\sigma, 1), \omega^{2}}^{ \pm} & =[(\sigma, 1), 0)], \quad[(1, \sigma), 0)], \\
\alpha_{(\tau, 1), 1}^{ \pm} & =[(\tau, 1), 0)]+[(\sigma \tau, 1), 0], \quad[(1, \tau), 0)]+[(1, \sigma \tau), 0], \\
\alpha_{(\tau, 1), \epsilon}^{ \pm} & =[(\tau, 1), 1)]+[(\sigma \tau, 1), 0], \quad[(1, \tau), 1)]+[(1, \sigma \tau), 0] .
\end{array}
$$


Consequently, we have the irreducible objects $\left[\alpha_{0}^{ \pm}\right],\left[\alpha_{1}^{ \pm}\right],\left[\alpha_{2}^{ \pm}\right]=\left[\alpha_{0}\right] \oplus\left[\alpha_{1}^{ \pm}\right]$, $\left[\alpha_{3}^{ \pm}\right]=\left[\alpha_{4}^{ \pm}\right]=\left[\alpha_{5}^{ \pm}\right],\left[\alpha_{6}^{ \pm}\right]=\left[\alpha_{6}^{ \pm(1)}\right] \oplus\left[\alpha_{6}^{ \pm(2)}\right]$ and $\left[\alpha_{7}^{ \pm}\right]=\left[\alpha_{6}^{ \pm(1)}\right] \oplus\left[\alpha_{7}^{ \pm(2)}\right]$. Moreover, $\left[\alpha_{1}^{+}\right]=\left[\alpha_{1}^{-}\right]$denoted henceforth by $\left[\alpha_{1}\right],\left[\alpha_{6}^{+(2)}\right]=\left[\alpha_{6}^{-(2)}\right]$ denoted from now by $\left[\alpha_{6}^{(2)}\right]$ and similarly $\left[\alpha_{7}^{(2)}\right]$. Thus ${ }_{M} \mathcal{X}_{M}^{0}=\left\{\alpha_{0}, \alpha_{1}, \alpha_{6}^{(2)}, \alpha_{7}^{(2)}\right\}$. Also the $\mathcal{X}^{ \pm}$-chiral systems are ${ }_{M} \mathcal{X}_{M}^{ \pm}=\left\{\alpha_{0}, \alpha_{1}, \alpha_{6}^{(2)}, \alpha_{7}^{(2)}, \alpha_{3}^{ \pm}, \alpha_{6}^{ \pm(1)}\right\}$. The corresponding modular invariant is $Z_{(22)}$ :

$$
Z_{(22)}=\left|\chi_{0}+\chi_{2}\right|^{2}+\left|\chi_{1}+\chi_{2}\right|^{2}+\left|\chi_{6}\right|^{2}+\left|\chi_{7}\right|^{2}
$$

(iv) Example: $H_{7}=\Delta\left(\mathbb{Z}_{3}\right)$.

The next case $H=H_{7}$ has 8 double cosets:

$$
\begin{gathered}
H(1,1)=H, H(1, \sigma) H=H(1, \sigma), H\left(1, \sigma^{2}\right) H=H\left(1, \sigma^{2}\right), H(1, \tau) H=\left(\mathbb{Z}_{3} \times \mathbb{Z}_{3}\right)(1, \tau), \\
H(\tau, 1) H=\left(\mathbb{Z}_{3} \times \mathbb{Z}_{3}\right)(\tau, 1), H(\tau, \tau) H=H(\tau, \tau), H(\sigma \tau, \tau) H, H\left(\sigma^{2} \tau, \tau\right) H,
\end{gathered}
$$

with corresponding stabilisers $\mathbb{Z}_{3}, \mathbb{Z}_{3}, \mathbb{Z}_{3}, 1,1, \mathbb{Z}_{3}, \mathbb{Z}_{3}, \mathbb{Z}_{3}$ respectively giving twenty irreducible objects in $\mathrm{Bun}^{H_{7}-H_{7}}(\Gamma)$. We decompose as $H$-H double cosets:

$$
\begin{aligned}
& H \Delta(1,1) \Delta H=H(1,1) H+H(\tau, \tau) H, \\
& H \Delta(\sigma, 1) \Delta H=H(1, \sigma) H+H(\tau, \sigma \tau) H+H(\sigma \tau, \tau) H+H\left(1, \sigma^{2}\right) H, \\
& H \Delta(\tau, 1) \Delta H=H(1, \tau) H+H(\tau, 1) H .
\end{aligned}
$$

Then $\alpha$-induction becomes:

$$
\begin{array}{ll}
\alpha_{1,1} & =[(1,1), 0)], \\
\alpha_{1, \epsilon}^{ \pm} & =[(1,1), 0)], \\
\alpha_{1, \pi}^{ \pm} & \left.=[(1,1), \omega)]+\left[(1,1), \omega^{2}\right)\right], \\
\alpha_{(\sigma, 1), 1}^{ \pm} & =[(\sigma, 1), 1)]+[(1, \sigma), 1)], \\
\alpha_{(\sigma, 1), \omega}^{ \pm} & \left.=[(\sigma, 1), \omega)]+\left[(1, \sigma), \omega^{2}\right)\right], \\
\alpha_{(\sigma, 1), \omega^{2}}^{ \pm} & \left.\left.=\left[(\sigma, 1), \omega^{2}\right)\right]+[(1, \sigma), \omega)\right], \\
\alpha_{(\tau, 1), 1}^{ \pm} & =[(\tau, 1), 1)], \quad[(1, \tau), 1], \\
\alpha_{(\tau, 1), \epsilon}^{ \pm} & =[(\tau, 1), 1)], \quad[(1, \tau), 1] .
\end{array}
$$

Consequently, we have the irreducible objects: $\left[\alpha_{0}^{ \pm}\right]=\left[\alpha_{1}^{ \pm}\right], \quad\left[\alpha_{2}^{ \pm}\right]=\left[\alpha_{2}^{ \pm(1)}\right] \oplus$ $\left[\alpha_{2}^{ \pm(2)}\right], \quad\left[\alpha_{3}^{ \pm}\right]=\left[\alpha_{3}^{ \pm(1)}\right] \oplus\left[\alpha_{3}^{ \pm(2)}\right],\left[\alpha_{4}^{ \pm}\right]=\left[\alpha_{4}^{ \pm(1)}\right] \oplus\left[\alpha_{4}^{ \pm(2)}\right], \quad\left[\alpha_{5}^{ \pm}\right]=\left[\alpha_{5}^{ \pm(1)}\right] \oplus$ $\left[\alpha_{5}^{ \pm(2)}\right], \quad\left[\alpha_{6}^{ \pm}\right]=\left[\alpha_{7}^{ \pm}\right]$, with $\left[\alpha_{0}^{ \pm}\right],\left[\alpha_{j}^{ \pm(i)}\right]$ and $\left[\alpha_{6}^{ \pm}\right]$irreducible sectors $(i=$ $1,2 ; j=2,3,4,5)$. The commutative neutral system as sectors is formed with nine automorphisms $\left[\alpha_{0}\right],\left[\alpha_{j}^{(i)}\right]$, with $i=1,2 ; j=2,3,4,5$ isomorphic to $\mathbb{Z}_{3} \times \mathbb{Z}_{3}$. Hence the system ${ }_{M} \mathcal{X}_{M}^{ \pm}={ }_{M} \mathcal{X}_{M}^{0} \cup\left\{\alpha_{6}^{ \pm}\right\}$, with the other fusion rules given by $\left[\alpha_{j}^{(i)}\right]\left[\alpha_{6}^{ \pm}\right]=\left[\alpha_{6}^{ \pm}\right]\left[\alpha_{j}^{(i)}\right]=\left[\alpha_{6}^{ \pm}\right], i=1,2 ; j=2,3,4,5$. The other nine irreducible objects are from the decomposition: 


$$
\alpha_{6}^{+} \alpha_{6}^{-}=\sum_{i, j}\left[\left(\tau, \sigma^{i} \tau\right), \omega^{j}\right] .
$$

The corresponding modular invariant is:

$$
Z_{5}=\left|\chi_{0}+\chi_{1}\right|^{2}+2\left|\chi_{2}\right|^{2}+2\left|\chi_{3}\right|^{2}+2\left|\chi_{4}\right|^{2}+2\left|\chi_{5}\right|^{2} .
$$

There are two further symmetric groups $H_{8}=\mathbb{Z}_{2} \times \mathbb{Z}_{2}$, and $H_{14}=\mathbb{Z}_{3} \times \mathbb{Z}_{3}$. (vi) Example: $H_{8}=\mathbb{Z}_{2} \times \mathbb{Z}_{2}$. Here with $H=H_{8}$, there are four double cosets:

$$
H(1,1) H=H, H(\sigma, 1) H, H(1, \sigma) H, H(\sigma, \sigma) H
$$

with stabilisers $\mathbb{Z}_{2} \times \mathbb{Z}_{2}, 1 \times \mathbb{Z}_{2}, \mathbb{Z}_{2} \times 1,1$ respectively so that there are nine irreducible objects in $\operatorname{Bun}^{H-H}(\Gamma)$. We decompose as $H-H$ double cosets:

$$
\begin{aligned}
& H \Delta(1,1) \Delta H=H(1,1) H+H(\sigma, \sigma) H, \\
& H \Delta(\sigma, 1) \Delta H=H(1, \sigma) H+H(\sigma, 1)+H(\sigma, \sigma) H, \\
& H \Delta(\tau, 1) \Delta H=H(1,1) H+H(1, \sigma) H+H(\sigma, 1)+H(\sigma, \sigma) H .
\end{aligned}
$$

Then $\alpha$-induction becomes:

$$
\begin{array}{ll}
\alpha_{1,1} & =[(1,1), 0)], \\
\alpha_{1, \epsilon}^{ \pm} & =[(1,1),(1,0))], \quad[(1,1),(0,1))], \\
\alpha_{1, \pi}^{ \pm} & =[(1,1), 0)]+[(1,1),(1,0)], \quad[(1,1), 0)]+[(1,1),(0,1)], \\
\alpha_{(\sigma, 1), 1}^{ \pm} & =[(\sigma, 1),(0,0)], \quad[(1, \sigma),(0,0)], \\
\alpha_{(\sigma, 1), \omega}^{ \pm} & =[(\sigma, 1),(0,0)], \quad[(1, \sigma),(0,0)], \\
\alpha_{(\sigma, 1), \omega^{2}}^{ \pm} & =[(\sigma, 1),(0,0)], \quad[(1, \sigma),(0,0)], \\
\alpha_{(\tau, 1), 1}^{ \pm} & =[(1,1), 0]+[(\sigma, 1), 0],[(1,1), 0]+[(1, \sigma), 0], \\
\alpha_{(\tau, 1), \epsilon}^{ \pm} & =[(1,1),(1,0)]+[(\sigma, 1),(1,0)],[(1,1),(0,1)]+[(1, \sigma),(0,0)] .
\end{array}
$$

So computing we get that ${ }_{M} \mathcal{X}_{M}^{ \pm}=\left\{\alpha_{0}, \alpha_{1}^{ \pm}, \alpha_{3}^{ \pm}\right\}$with $\left[\alpha_{2}^{ \pm}\right]=\left[\alpha_{0}\right] \oplus$ $\left[\alpha_{1}^{ \pm}\right], \quad\left[\alpha_{5}^{ \pm}\right]=\left[\alpha_{4}^{ \pm}\right]=\left[\alpha_{3}^{ \pm}\right],\left[\alpha_{6}^{ \pm}\right]=\left[\alpha_{0}\right] \oplus\left[\alpha_{3}^{ \pm}\right],\left[\alpha_{7}^{ \pm}\right]=\left[\alpha_{1}^{ \pm}\right] \oplus\left[\alpha_{3}^{ \pm}\right]$. The sectors of ${ }_{M} \mathcal{X}_{M}^{ \pm}$are $\widehat{S_{3}}$. The corresponding modular invariant is

$$
Z_{44}=\left|\chi_{0}+\chi_{2}+\chi_{6}\right|^{2}
$$

(vi) Example: $H=H_{14}=\mathbb{Z}_{3} \times \mathbb{Z}_{3}$.

Taking $H=H_{14}$, we have four double cosets:

$$
H(1,1) H=H, H(\tau, 1) H, H(1, \tau) H, H(\tau, \tau) H,
$$

all with stabilisers $H$ so that there are 36 irreducible objects in $\operatorname{Bun}^{H-H}(\Gamma)$. We decompose as $H$ - $H$ double cosets: 


$$
\begin{aligned}
& H \Delta(1,1) \Delta H=H(1,1) H+H(\tau, \tau) H, \\
& H \Delta(\sigma, 1) \Delta H=H(1,1) H+H(\tau, \tau) H, \\
& H \Delta(\tau, 1) \Delta H=H(1, \tau) H+H(\tau, 1) H .
\end{aligned}
$$

Then $\alpha$-induction becomes:

$$
\begin{array}{ll}
\alpha_{1,1} & =[(1,1), 1)], \\
\alpha_{1, \epsilon}^{ \pm} & =[(1,1), 1], \\
\alpha_{1, \pi}^{ \pm} & =2[(1,1), 1], \\
\alpha_{(\sigma, 1), 1}^{ \pm} & =[(1,1),(\omega, 1)]+\left[(1,1),\left(\omega^{2}, 1\right)\right], \quad[(1,1),(1, \omega)]+\left[(1,1),\left(1, \omega^{2}\right)\right], \\
\alpha_{(\sigma, 1), \omega}^{ \pm} & =[(1,1),(\omega, 1)]+\left[(1,1),\left(\omega^{2}, 1\right)\right], \quad[(1,1),(1, \omega)]+\left[(1,1),\left(1, \omega^{2}\right)\right], \\
\alpha_{(\sigma, 1), \omega^{2}}^{ \pm} & =[(1,1),(\omega, 1)]+\left[(1,1),\left(\omega^{2}, 1\right)\right], \quad[(1,1),(1, \omega)]+\left[(1,1),\left(1, \omega^{2}\right)\right], \\
\alpha_{(\tau, 1), 1}^{+} & =[(\tau, 1),(1,1)]+[(\tau, 1),(\omega, 1)]+\left[(\tau, 1),\left(\omega^{2}, 1\right)\right], \\
\alpha_{(\tau, 1), 1}^{-} & =[(1, \tau),(1,1)]+[(1, \tau),(1, \omega)]+\left[(1, \tau),\left(1, \omega^{2}\right)\right], \\
\alpha_{(\tau, 1), \epsilon}^{+} & =[(\tau, 1),(1,1)]+[(\tau, 1),(\omega, 1)]+\left[(\tau, 1),\left(\omega^{2}, 1\right)\right], \\
\alpha_{(\tau, 1), \epsilon}^{-} & =[(1, \tau),(1,1)]+[(1, \tau),(1, \omega)]+\left[(1, \tau),\left(1, \omega^{2}\right)\right] .
\end{array}
$$

Hence we have the chiral system:

$$
{ }_{M} \mathcal{X}_{M}^{+}=\left\{\alpha_{0}, \alpha_{3}^{+(1)}, \alpha_{3}^{+(2)}, \alpha_{6}^{+(1)}, \alpha_{6}^{+(2)}, \alpha_{6}^{+(3)}\right\}
$$

with $\left[\alpha_{0}\right]=\left[\alpha_{1}^{+}\right],\left[\alpha_{2}^{+}\right]=2\left[\alpha_{0}\right],\left[\alpha_{3}^{+}\right]=\left[\alpha_{4}^{+}\right]=\left[\alpha_{5}^{+}\right]=\left[\alpha_{3}^{+(1)}\right] \oplus\left[\alpha_{3}^{+(2)}\right]$, $\left[\alpha_{7}^{+}\right]=\left[\alpha_{6}^{+}\right]=\left[\alpha_{6}^{+(1)}\right] \oplus\left[\alpha_{6}^{+(2)}\right] \oplus\left[\alpha_{6}^{+(3)}\right]$, and similarly

$$
{ }_{M} \mathcal{X}_{M}^{-}=\left\{\alpha_{0}, \alpha_{3}^{-(1)}, \alpha_{3}^{-(2)}, \alpha_{6}^{-(1)}, \alpha_{6}^{-(2)}, \alpha_{6}^{-(3)}\right\} .
$$

We can conclude that ${ }_{M} \mathcal{X}_{M}^{ \pm}$is as sectors $S_{3}$. The corresponding modular invariant is $Z_{33}$ :

$$
Z_{33}=\left|\chi_{0}+\chi_{1}+2 \chi_{3}\right|^{2} .
$$

Acknowledgement. This work was supported by the EU Network QSNG in Quantum Spaces - Noncommutative Geometry and the EPSRC Network ABC-KLM in Algebraic Geometry, Boundary Conformal Field Theory and Noncommutative Geometry. I thank Daniel Hoyt for assistance in some of the computations and discusssions at an early stage of this work, Ryszard Nest for discussions throughout and to Paulo Pinto for a careful reading of the manuscript. I am grateful to ANU Canberra, Copenhagen University, DIAS Dublin, IST Lisbon, Oxford University, Tor Vergata Rome, the University of Oslo and particularly Alan Carey, Ryszard, Nest, Denjoe O'Connor, Paulo Pinto, Keith Hannabuss, Roberto Longo, John Roberts, Ola Bratteli, Erling Stormer for their warm hospitality during visits when much of this work was done. 


\section{References}

[AC] Altschuler, A., Coste, A.: Quasi-quantum groups, knots, three manifolds and topological field theory. Commun. Math. Phys., 150, 83-107 (1992).

[ACM] Altschuler, A., Coste, A., Maillard, J-M.: Representation theory of twisted group double. Ann. Fond. Louis de Broglie. 29, 681-694 (2004).

[A] Atiyah, M.F.: K-theory past and present. Sitzungsberichte der Berliner Mathematischen Gesellschaft, 411-417, Berliner Math. Gesellschaft, Berlin, 2001. math.KT/0012213

[AS] Atiyah, M.F., Segal, G.B.: Twisted K-theory. math.KT/0407054

[BK] Bakalov. B., Kirillov, A.: Lectures on tensor cateogries and modular functors. University Lecture Series, vol 21, American Mathematical Society, Providence, R.I. 2001

[B] Baumgärtel, H.: Operatoralgebraic methods in quantum field theory. Berlin: Akademie Verlag, 1995

[BE1] Böckenhauer, J., Evans, D.E.: Modular invariants, graphs and $\alpha$-induction for nets of subfactors. I. Commun. Math. Phys., 197, 361-386 (1998); II Commun. Math. Phys., 200, 57-103 (1999); III Commun. Math. Phys. 205, 183-228 (1999)

[BE2] Böckenhauer, J., Evans, D.E.: Modular invariants from subfactors: Type I coupling matrices and intermediate subfactors. Commun. Math. Phys., 213, 267-289 (2000)

[BE3] Böckenhauer, J., Evans, D.E.: Modular invariants from subfactors, in Quantum symmetries in theoretical physics and mathematics (Bariloche, 2000), pp 95-131, Contemp. Math., 294, Amer. Math. Soc., Providence, RI, 2002

[BE4] Böckenhauer, J., Evans, D.E.: Modular invariants and subfactors. Mathematical physics in mathematics and physics (Siena, 2000), 11-37, Fields Inst. Commun., 30, Amer. Math. Soc., 2001.

[BEK1] Böckenhauer, J., Evans, D.E., Kawahigashi, Y.: On $\alpha$-induction, chiral generators and modular invariants for subfactors. Commun. Math. Phys., 208, 429-487 (1999)

[BEK2] Böckenhauer, J., Evans, D.E., Kawahigashi, Y.: Chiral structure of modular invariants for subfactors. Commun. Math. Phys., 210, 733-784 (2000)

[BEK3] Böckenhauer, J., Evans, D.E., Kawahigashi, Y.: Longo-Rehren subfactors arising from $\alpha$-induction. Publ. RIMS, Kyoto Univ., 37, 1-35 (2001)

[BCMMS] Bouwknegt, P., Carey, A.L., Mathai, V., Murray, M.M., Stevenson, D.: Twisted K-theory and K-theory of bundle gerbes. hep-th/0106194

[C] Cardy, J.: Fusion rules and the Verlinde formula. Nucl. Phys. B, 324, 581-596 (1989)

[CGP] Coste, A., Gannon, T., Philippe, R.: Finite group modular data. Nucl. Phys. B, 581, 679-717 (2000)

[DCVV] Dijkgraaf, R.; Cumrun, V.; Verlinde, E.; Verlinde, H.: The operator algebra of orbifold models. Commun. Math. Phys., 123, 485-526 (1989)

[DPR] Dijkgraaf, R., Pasquier, V., Roche, P.: Quasi-quantum groups related to orbifolds models. Modern quantum field theory (Bombay, 1990), 375-383, World Sci. Publishing, River Edge, NJ, 1991.

[DD] Dixmier, J., Douady, A.: Champs continus d'espaces hilbertiens et de C*algebras. Bull. Soc. Math. France, 91, 227-284 (1963) 
[E1] Evans, D.E.: Fusion rules of modular invariants. Rev. Math. Phys., 14, 709-732 (2002)

[E2] Evans, D.E.: Critical phenomena, modular invariants and operator algebras. Operator algebras and mathematical physics (Constanţa 2001, J. Cuntz, G.A. Elliott, S. Stratila et al. eds.), The Theta Foundation, Bucharest, 2003

[E3] Evans, D.E.: Modular invariant partition functions in statistical mechanics, conformal field theory and their realisation by subfactors. Proceedings Congress of International Association of Mathematical Physics, Lisbon 2003, to appear.

[EK] Evans, D. E., Kawahigashi, Y.: Quantum symmetries on operator algebras. Oxford University Press (1998)

[EP1] Evans, D.E., Pinto, P.R.: Modular invariants and their fusion rules in Advances in Quantum Dynamics (Mount Holyoke, 2002), pp. 119-130, Contemp. Math. 335, Amer. Math. Soc., Providence, RI, 2003

[EP2] Evans, D.E., Pinto, P.R.: Subfactor realisation of modular invariants. Commun. Math. Phys., 237, 309-363 (2003)

[EP3] Evans, D.E., Pinto, P.R.: Modular invariants and the double of the Haagerup subfactor. Sinaia Proceedings, to appear.

[EP4] Evans, D.E., Pinto, P.R.: Subfactor realisation of modular invariants, II.

[F1] Freed, D.S.: Higher algebraic structures and quantisation. Commun. Math. Phys., 159, 343-398 (1994).

[F2] Freed, D.S.: The Verlinde algebra is twisted equivariant K-theory. Turkish J. Math., 25, 159-167 (2001).

[F3] Freed, D.S.: Twisted K-theory and loop groups. Proceedings of the International Congress of Mathematicians, Vol. III (Beijing, 2002), 419-430, Higher Ed. Press, Beijing, 2002.

[F4] Freed, D.S.: K-theory in quantum field theory. Current developments in mathematics, 2001, 41-87, Int. Press, Somerville, MA, 2002.

[FHT] Freed, D.S., Hopkins, M.J., Teleman, C.: Twisted equivariant K-theory with complex coefficients. math-ph/020631

[FQ] Freed, D.S., Quinn, F.: Chern Simmons theory with finite gauge group. Commun. Math. Phys., 156, 435-472 (1993)

[FZ] di Francesco, P.; Zuber, J.B.: $S U(N)$ lattice integrable models associated with graphs. Nucl. Phys. B, 338, 602-646 (1990)

[FG] Fröhlich, J., Gabbiani, F.: Operator algebras and conformal field theory. Commun. Math. Phys., 155, 569-640 (1993)

[G] Gannon, T.:Modular data: the algebraic combinatorics of conformal field theory. hep-th/0103044

[I1] Izumi, M.: The structure of sectors associated with Longo-Rehren inclusions, I. General theory. Commun. Math. Phys., 213, 127-179 (2000)

[I2] Izumi, M.: The structure of sectors associated with Longo-Rehren inclusions, II. Examples. Rev. Math. Phys., 13, 603-674 (2001)

[KI] Izumi, M., Kosaki, H.: On the subfactor analogue of the second cohomology. Preprint, Kyoto-Math 2002-01

[J1] Jones, V. F. R.: An invariant for group actions. Alg`ebres d'op`erateurs (Sèm., Les Plans-sur-Bex, 1978), pp. 237-253, Lecture Notes in Math., 725, Springer, Berlin, 1979.

[J2] Jones, V.F.R.: Index for subfactors Invent. Math., 72, 1-25(1983) 
[KT] Katayama, Y., Takesaki, M.: Outer actions of a countable discrete amenable group on an AFD factor. Advances in quantum dynamics (South Hadley, MA, 2002), 163-171, Contemp. Math., 335, Amer. Math. Soc., Providence, RI, 2003.

[KSW] Kawahigashi, Y., Sato, N., Wakui, M. (2+1)-dimensional topological quantum field theory from subfactors and Dehn surgery formula for 3manifold invariants. Preprint math.OA/0208238

[KO] Kirillov, A., Jr.; Ostrik, V.: On a $q$-analogue of the McKay correspondence and the ADE classification of $\hat{\mathfrak{s}} l_{2}$ conformal field theories. Adv. Math., 171, 183-227 (2002)

[KY] Kosaki, H., Yamagami, S.: Irreducible bimodules associated with crossed product algebras. Internat. J. Math., 3, 661-676 (1992)

[KMY] Kosaki, H., Munemasa, A., Yamagami, S.: On fusion algebras associated to finite group actions Pacific J. Math., 177 269-290 (1997)

[L] Longo, R.: Index of subfactors and statistics of quantum fields, II. Commun. Math. Phys., 130, 285-309 (1990)

[LR] Longo, R., Rehren, K.-H.: Nets of subfactors. Rev. Math. Phys., 7, 567597 (1995)

[M] Mason, G.: The quantum double of a finite group and its role in conformal field theory. Groups '93 Galway/St. Andrews, Vol. 2, 405-417, London Math. Soc. Lecture Note Ser., 212, Cambridge Univ. Press, Cambridge, 1995

[O1] Ocneanu, A. Paths on Coxeter diagrams: From Platonic solids and singularities to minimal models and subfactors, in Lectures on operator theory, Rajarama Bhat, B.V. et al. (eds.) (Notes recorded by S. Goto), The Fields Institute Monographs, Providence, Rhode Island: AMS publications 2000, pp. 243-323

[O2] Ocneanu, A.: The classification of subgroups of quantum $S U(N)$, in Quantum symmetries in theoretical physics and mathematics (Bariloche, 2000), Contemp. Math., 294, Amer. Math. Soc., Providence, RI, 2002, pp 133159

[Ost1] Ostrik, V.:Module categories, weak Hopf algebras and modular invariants. Transform. Groups, 8, 177- 206 (2003) or math.QA/0111139

[Ost2] Ostrik, V.: Module categories for quantum doubles of finite groups. Inter. Math. Research. Notices, 27, 1507-1520(2003), math.QA/0202130

[R] Rieffel, M. Morita equivalence for operator algebras. Operator algebras and applications, Part I (Kingston, Ont., 1980), pp. 285-298, Proc. Sympos. Pure Math., 38, Amer. Math. Soc., Providence, R.I., 1982.

[SW] Sato, N., Wakui, M. Computations of Turaev-Viro-Ocneanu invariants of 3-manifolds from subfactors. J. Knot Theory Ramifications, 12, 543-574 (2003)

[S] Sutherland, C.: Cohomology and extensions of von Neumann algebras. I, II. Publ. RIMS, Kyoto Univ., 16, 105-133 , 135-174 (1980)

[T] Turaev, V.G. Quantum invariants of knots and 3-manifolds. in de Gruyter Studies in Mathematics, vol 18. Walter de Gruyter, Berlin, 1994

[V] Verlinde, E.: Fusion rules and modular transformations in 2D conformal field theory. Nucl. Phys. B, 300, 360-376 (1988)

[W1] Wassermann, A.: Operator algebras and conformal field theory III: fusion of positive representations of LSU(n) using bounded operators. Invent. Math., 33, 467-538 (1998) 
[W2] Wassermann, A.: Automorphic represenations of real and p-adic Lie groups. Noncommutative Geometry, Oberwolfach Report No. 45/2004.

[X] Xu, F.: New braided endomorphisms from conformal inclusions. Commun. Math. Phys., 192, 349-403 (1998) 